\title{
Distributed Demand Side Management with Stochastic Wind Power Forecasting
}

\author{
Paolo Scarabaggio, Student Member, IEEE, Sergio Grammatico, Senior Member, IEEE, \\ Raffaele Carli, Member, IEEE, and Mariagrazia Dotoli, Senior Member, IEEE
}

\begin{abstract}
In this paper, we propose a distributed demand side management (DSM) approach for smart grids taking into account uncertainty in wind power forecasting. The smart grid model comprehends traditional users as well as active users (prosumers). Through a rolling-horizon approach, prosumers participate in a DSM program, aiming at minimizing their cost in the presence of uncertain wind power generation by a game theory approach. We assume that each user selfishly formulates its grid optimization problem as a noncooperative game. The core challenge in this paper is defining an approach to cope with the uncertainty in wind power availability. We tackle this issue from two different sides: by employing the expected value to define a deterministic counterpart for the problem and by adopting a stochastic approximated framework. In the latter case, we employ the sample average approximation technique, whose results are based on a probability density function (PDF) for the wind speed forecasts. We improve the PDF by using historical wind speed data, and by employing a control index that takes into account the weather condition stability. Numerical simulations on a real dataset show that the proposed stochastic strategy generates lower individual costs compared to the standard expected value approach.
\end{abstract}

Index Terms-Smart grid, Demand side management, Sample average approximation, Stochastic optimization.

\section{INTRODUCTION}

$\mathbf{T}$ HE CONTEMPORARY energy landscape includes a limited number of industries that concentrate the electricity production in some fossil and nuclear power plants. Moreover, the electricity distribution system is still unidirectional and passive. This architecture makes it difficult to profitably coordinate supply and demand. Indeed, most of the time, suppliers have to operate expensive ancillary plants in order to satisfy the changing power demand, with a significant environmental impact. Moreover, the growing demand for energy drives the formulation of plans to expand and upgrade the contemporary electricity grids. However, energy sustainability is currently a worldwide concern, making it difficult to use old paradigms for these upgrades.

In this context, smart grids find their place in leading to new features in the management of the electricity network.

The work of S. Grammatico is partially supported by NWO under projects OMEGA (613.001.702) and P2P-TALES (647.003.003), and by the ERC under project COSMOS (802348). The work of M. Dotoli is partially supported by Italian University and Research Ministry under project RAFAEL (National Research Program, contract n. ARS01-00305).

P. Scarabaggio, R. Carli, and M. Dotoli are with the Department of Electrical and Information Engineering of the Polytechnic of Bari, Italy. (email: \{paolo.scarabaggio, raffaele.carli, mariagrazia.dotoli\}@poliba.it).

S. Grammatico is with the Delft Center for Systems and Control, TU Delft, The Netherlands. (e-mail: s.grammatico@tudelft.nl).
Among the basic functionalities of smart grids, there are demand side management (DSM), distributed generation (DG), and distributed storage (DS). As a result, smart grids allow balancing and coping with energy peaks, improving efficiency, security, and quality of the power distribution system [1]. Furthermore, one of the most important advantages of smart grids is their potential to efficiently integrate renewable energy sources (RES) in the distribution system. In fact, in the traditional configuration system, this integration is difficult due to the highly intermittent and stochastic behavior of weather conditions.

\section{A. Literature review}

There is a considerable amount of scientific literature on smart grids, and various approaches have been proposed to implement DSM programs [2]-[5].

A growing body of literature studies the use of game theory in DSM. Advantages of such an approach are in its ability to straightforwardly model the selfish nature of users and in its capacity to model decentralized or distributed control strategies, which are particularly effective in the case of largescale systems [6], [7]. For instance, in [6], the authors propose a Stackelberg game between the electricity provider and users: the model is able to maximize the profit of the distribution provider and the welfare of the users. However, the presented approach is developed only for the deterministic case and does not consider the presence of RESs. In [7], a pricing strategy encourages the optimal generation and storage by the users. The study focuses only on the day-ahead optimization problem, and unfortunately it does not include RESs. In addition, in [8] the authors present an optimization algorithm for the scheduling of electrical activities of a microgrid, where users are connected to a distributor and are allowed to exchange renewable energy produced by individually owned distributed resources. However, such a renewable energy production is modeled in a deterministic way, disregarding the presence of uncertainty.

Essentially, the uncertainty in renewable energy production makes it hard to choose the optimal scheduling strategy of a smart grid. Therefore, DSM techniques can help mitigating this variability. In the related literature two approaches are typically employed: updating the RES forecasts (e.g., using a rolling-horizon approach), or employing probability distributions. In the first class of approaches, we recall a noncooperative model that includes a wind power source presented in [9]. In the model, all the potential uncertain 
variables are supposed to be deterministic, and a shrinkinghorizon approach is used to handle their possible variations during the day.

Within the second class of approaches, we recall [10], where the authors propose a stochastic optimization model for the day-ahead energy scheduling, which incorporates DSM for managing the variability of RES. The proposed stochastic day-ahead algorithm considers random outages of system components and forecast errors for the RES. A Monte Carlo simulation creates multiple scenarios for representing possible realizations of uncertainty, and a mixed-integer linear problem is employed to solve the resulting stochastic problem. In [11], a risk-averse strategy for islanded microgrids with a high share of RES is presented. A sampling-based Monte Carlo forecast is chosen to handle RES variability, generating a collection of independent scenarios with the same probability. Other models represent uncertainty in the wind power availability, where wind power prediction errors are typically assumed to follow a Gaussian probability distribution. On the other hand, a number of studies suggest the use of the Weibull and Rayleigh distribution to characterize the wind speed [12], [13]. In [14], a Weibull distribution is used to forecast the wind speed, and a nonlinear function is employed to transform it into a power probability density function (PDF) for the power output. The objective function considers the reserve cost for overestimation and penalty cost for underestimation of RES power availability. The same approach is used in [15], where the authors investigate the effects of renewable energy integration on the power system from a stochastic point of view. In [16], the authors implement a stochastic programming strategy, by using a historical curve for the wind speed scenarios, and by validating the results using the wind speed forecasts. However, approaches in [10]-[16] rely on a centralized framework, that is questionable in case of large scale systems. Other references [17], [18] propose the use of a normal distribution aimed at considering the wind speed variability. For instance, in [19], a Monte Carlo simulation is employed to generate a scenario tree to predict wind power availability. Moreover, a stochastic planning approach based on a Monte Carlo method is proposed in [20] to model the uncertain wind behavior.

\section{B. Contribution}

In this paper, we propose a smart grid model that comprises a central unit and several interconnected demand-side users with a wind turbine. Users can be merely energy consumers, or they can participate actively in the grid optimization process. We implement a DSM model where the central unit updates the energy price based on the aggregate load and the wind power availability. We assume that each active user acts selfishly, trying to minimize his total expense by updating his DG and DS strategies. However, choices have to be made by users respecting their local preference and the overall grid constraints. To take into account the selfish nature of users, we model the resulting energy scheduling problem as a Nash game, extending the approach originally developed in [7] for the day-ahead deterministic scheduling case and subsequently modified in [9] using a shrinking-horizon technique in a deterministic and semi-decentralized setting. More precisely, we present a novel receding-horizon DSM strategy in a stochastic and semi-decentralized setting.

In this context, we consider the uncertainty in wind power availability, and our policy to manage this significant issue is twofold. Firstly, we model the DSM model in a rolling-horizon fashion, considering updatable wind speed forecasts. Secondly, we propose a stochastic distributed optimization strategy. We propose a framework where each user could consider a Weibull distribution based on historical wind speed data and a Gaussian distribution to locally model the uncertainty in the wind speed forecasts. Furthermore, we firstly apply to the smart grid context an instability index that evaluates the overall atmospheric conditions and modifies the latter distribution. Consequently, each user could generate a PDF for the wind power availability by adopting an approximated nonlinear relation between wind speed and power. By merging the historical and forecast PDF, we address the cases where the wind speed forecasts are particularly uncertain. We obtain a number of samplings from the aforementioned PDFs and use the sample average approximation (SAA) to generate an averaged cost function that takes into account several different wind power availability scenarios.

Finally, we show the effectiveness of the proposed mechanism on a realistic case study, using real forecasts and real historical measured data. In particular, we examine the advantage that a single user obtains by adopting the proposed stochastic approach with respect to other competitors that employ a deterministic counterpart for the problem.

\section{Positioning of our contribution within the related literature}

Summing up, many attempts have been made to design DSM strategies for smart grid management [6], [7]. However, the majority of works focuses only on the day-ahead optimization problem, while considering the supply side exclusively. In our work, instead, we propose a model that includes a wind turbine, and we perform a long-term analysis employing real wind speed forecasts. Moreover, we validate the results with real on-site measured wind speed data.

Regarding the RES presence in the grid, the majority of the studies in the related literature, see for instance [3], [8], [9], considers the RES power availability as a deterministic variable, disregarding the stochastic nature of these sources. The few approaches considering uncertainty in wind power availability typically define a discrete PDF for the possible outcomes or to assume the wind power prediction errors to follow a Gaussian probability distribution [10], [11]. However, these approaches rely on a centralized framework which has evident computational and privacy limitation. In addition, one of the significant drawbacks of these methods is the incorrect representation of wind power uncertainty due to the nonlinearity in the wind power generation process. Hence, to overcome this issue, in this work we consider the wind power availability as a stochastic variable, and describe its uncertainty by defining a PDF based on real forecast and real historical data, assessing all the parameters values. Moreover, we use 
this PDF to perform a stochastic programming approach and improve the performance of the model.

\section{Paper organization}

The rest of this work is structured as follows. In Section II, we present the smart grid model, with the demand side, generation, and storage models; lastly, we define the cost and the pricing market. Section III comprehends the wind power characterization, a model to obtain a PDF for the wind power, and a methodology to combine information coming from the forecasts and the historical data. In Section IV, we define the non-cooperative game, and the algorithm to reach an equilibrium. In Section V, we show the numerical results of the simulation. Finally, in Section VI, we conclude the paper and discuss an outlook for future work.

\section{SMART GRID MODEL}

The focus of this section is to present the smart grid model, where we consider as a starting point the smart grid introduced in [7]. The model in [7] considers a day-ahead optimization process for a group of active users regulated by a central unit. The active users modify their energy scheduling profile aiming at decreasing their total cost by using some distributed energy sources or energy storage devices. A related approach is employed in [9], improving the model in [7] including a RES in the supply side and implementing a shrinking-horizon approach on a daily base to cope with the uncertainty in wind power availability. The model includes an algorithm to reach an equilibrium between the active users while respecting the overall grid constraints.

For the purpose of a long-time simulation, we modify the approaches presented in [7] and [9] considering a recedinghorizon strategy on an hourly basis. We suppose that the control and the prediction horizon have a fixed length $H$, and they are moving ahead at every time slot (hence, eventually straddling two days). Moreover, we define the control horizon at the generic time slot $k$ as $\mathcal{H}=\{k, \ldots, k+H-1\}$.

The scheme of the considered smart grid is represented in Fig. 1. The smart grid is composed of a central unit, and several users that share a wind turbine. Users are also connected to the distribution system from/to which they can buy or sell electricity, and they are also connected to a central unit, which is able to keep the overall smart grid electricity consumption inside a fixed range. In our setting, the central unit broadcasts the hourly price coefficients and forecasts the wind speed for the wind turbine location. Each active user has an energy controller that decides a consumption, generation and storage strategy, based on its devices' status, the wind speed forecasts, and the aggregate grid load.

\section{A. Flexible demand-side model}

We consider a group of demand side users $\mathcal{D}$, divided into two subsets of passive $\mathcal{P}$ and active $\mathcal{N}$ users (prosumers), where $\mathcal{D}=\mathcal{P} \cup \mathcal{N}$ and $\mathcal{P} \cap \mathcal{N}=\varnothing$, with cardinality $D$, $P$ and $N$ respectively. Each user $i \in \mathcal{D}$ has a per-slot noncontrollable energy consumption $e_{i}(h), \forall, h \in \mathcal{H}$. Let us define the non-controllable energy consumption scheduling vector $\boldsymbol{e}_{i}=\left(e_{i}(h)\right)_{h \in \mathcal{H}}$. Passive users $i \in \mathcal{P}$ are merely energy consumers, while active users $i \in \mathcal{N}$ participate in the grid optimization process by modifying their, controllable energy consumption, distributed production or storage strategies. We assume that each active user is connected bidirectionally to the power grid, and to a communication infrastructure that enables a two-way communication between the user's energy controller and the central unit. In addition to the noncontrollable energy consumption, all active users have a perslot controllable energy consumption $d_{i}(h)$ for $h \in \mathcal{H}$ (e.g., a heating system or a boiler), that they can modify based on the market condition. We assume a first constraint on the controllable energy consumption as follows:

$$
d_{i}^{\min } \leq d_{i}(h) \leq d_{i}^{\max }, \quad \forall h \in \mathcal{H}, \forall i \in \mathcal{N} .
$$

Moreover, we assume that the controllable energy demand must be satisfied on a daily base, therefore, let us include an additional constraint on the daily energy demand. Since in our model the control horizon can straddle on two days, it is useful to define $\mathcal{H}_{1}$ as the set that comprehends the remaining time slots of the preceding day, and consequently, $\mathcal{H}_{2}$ the time slots of the subsequent day included in the control horizon. By naming $\bar{h}$ the last time slot of the preceding day, we can write more formally:

$$
\begin{gathered}
\mathcal{H}_{1}=[k, \bar{h}] \cap \mathbb{Z}, \quad \mathcal{H}_{2}=[\bar{h}+1, k+H-1] \cap \mathbb{Z} \\
\sum_{h \in \mathcal{H}_{1}} d_{i}(h)=\xi_{i, 1}^{\text {left }}, \quad \sum_{h \in \mathcal{H}_{2}} d_{i}(h)=\xi_{i, 2}, \quad \forall i \in \mathcal{N}
\end{gathered}
$$

where $\xi_{i, 2}$ is the controllable energy demand in the second day, and $\xi_{i, 1}^{\text {left }}$ is the remaining controllable energy demand in the first day, i.e., the remaining part of the daily controllable energy demand of the first day that must be satisfied by the end of the day. Let us define for each prosumer $i \in \mathcal{N}$ the controllable energy consumption scheduling vector $\boldsymbol{d}_{i}=\left(d_{i}(h)\right)_{h \in \mathcal{H}}$ and a set of feasible strategies:

$$
\boldsymbol{\Omega}_{\boldsymbol{d} i}=\left\{\boldsymbol{d}_{i} \in \mathbb{R}_{\geq 0}^{H} \mid(1),(3) \text { hold }\right\}, \quad \forall i \in \mathcal{N} .
$$

Moreover, a prosumer can be either a dispatchable energy producer $i \in \mathcal{G}$ with a per-slot energy generation $g_{i}(h)$, and/or an energy storage user $i \in \mathcal{S}$ with per-slot energy storage profile $s_{i}(h)$. Note that in general $\mathcal{G} \cap \mathcal{S} \neq \varnothing$ and $\mathcal{G} \cup \mathcal{S}=\mathcal{N}$. The per-slot load profile $l_{i}(h), \forall h \in \mathcal{H}, \forall i \in \mathcal{D}$ is hence:

$$
l_{i}(h)= \begin{cases}e_{i}(h) & \text { if } i \in \mathcal{P} \\ e_{i}(h)+d_{i}(h)-g_{i}(h)+s_{i}(h) & \text { if } i \in \mathcal{N}\end{cases}
$$

which expresses the energy flow between the grid and user $i \in \mathcal{D}$. For every user $i \in \mathcal{D}$ let us then define for the control horizon $\mathcal{H}$, the energy load scheduling vector $\boldsymbol{l}_{i}=\left(l_{i}(h)\right)_{h \in \mathcal{H}}$. Obviously, for all the passive users $i \in \mathcal{P}$ it holds $\boldsymbol{l}_{i}=\boldsymbol{e}_{i}$.

\section{B. Flexible energy generation model}

Let us consider the group of prosumers $i \in \mathcal{G}$ equipped with dispatchable energy devices (e.g., internal combustion engines, gas turbines, or fuel cells). These active users can thus: produce energy to satisfy their demand, charge their 


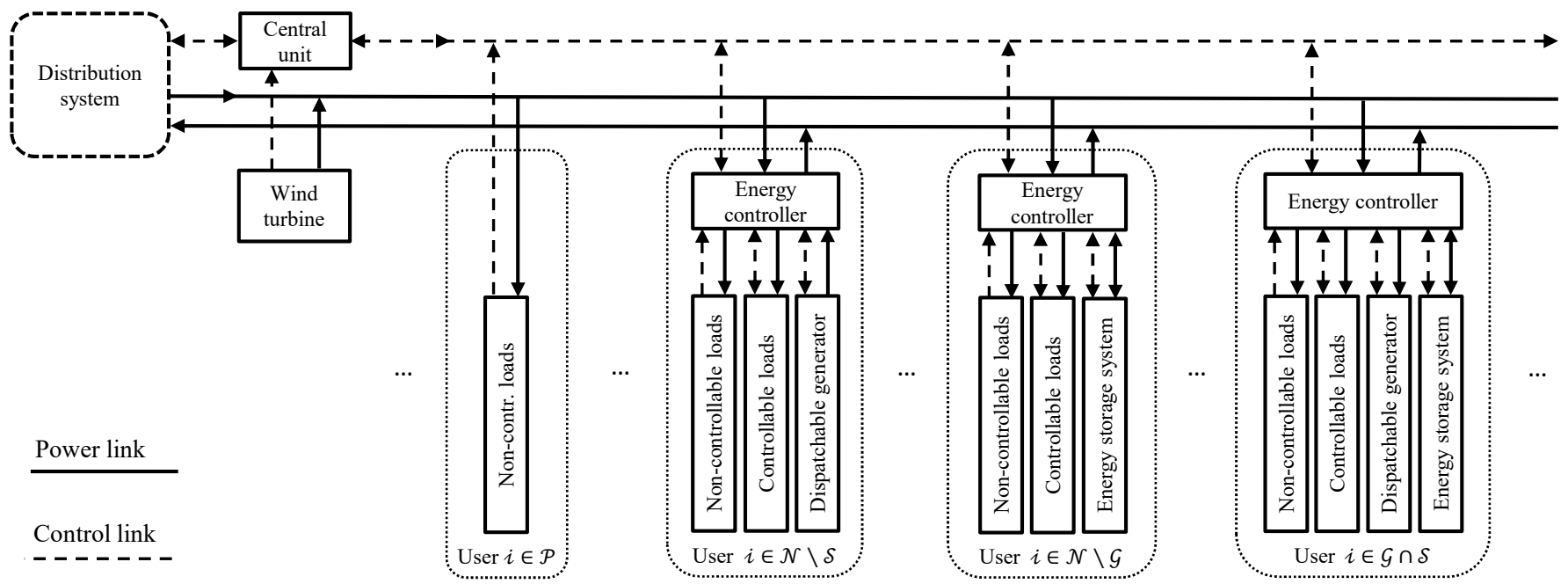

Fig. 1. Scheme of energy flows and connections in the smart grid.

battery, or sell to the grid during the peak time slots when the energy request is higher. We assume that each energy producer is subject to variable production costs (e.g., fuel cost, maintenance) and fixed cost. Let us define an energy production cost function $W_{i}\left(g_{i}(h)\right)$ for all the users $i \in \mathcal{G}$, comprehending the variable production costs, i.e., the fuel and the maintenance costs. We assume that $W_{i}$ is linear with a coefficient $\eta_{i}$, i.e., $W_{i}\left(g_{i}(h)\right)=\eta_{i} g_{i}(h)$. These prosumers are interested in optimizing their production strategies to obtain the highest advantage while respecting their local preferences. We assume so a first constraint in the generation capacity; besides a non-negative minimum, a maximum per-slot energy generation can take into account the devices' technological restrictions:

$$
0 \leq g_{i}(h) \leq g_{i}^{\max }, \quad \forall h \in \mathcal{H}, \forall i \in \mathcal{G} .
$$

In order to prevent the device's overuse, we include additional constraints on the maximum production over $H$ consecutive time slots:

$$
\sum_{z=h-H+1}^{h} g_{i}(z) \leq \psi_{i}, \quad \forall h \in \mathcal{H}, \forall i \in \mathcal{G}
$$

where $\psi_{i}$ is the maximum amount of energy that active user $i \in \mathcal{G}$ can generate in a period of $H$ time slots. Note that, in (7) we also consider the energy generated in the time slots that precede the control horizon; therefore, these constraints depend on the previously implemented strategies.

Obviously, at each time slot $h$ we have that $g_{i}(h)=0$ for all the prosumers $i \notin \mathcal{G}$. Let us define for each producer $i \in \mathcal{G}$ an energy production scheduling vector $\boldsymbol{g}_{i}=\left(g_{i}(h)\right)_{h \in \mathcal{H}}$ and a set of feasible strategies:

$$
\boldsymbol{\Omega}_{\boldsymbol{g}_{i}}=\left\{\boldsymbol{g}_{i} \in \mathbb{R}_{\geq 0}^{H} \mid(6),(7) \text { hold }\right\}, \quad \forall i \in \mathcal{G} .
$$

\section{Flexible energy storage model}

Let us now model the group of energy storage devices (e.g., batteries, flywheels, fuel cells) owned by the prosumers $i \in \mathcal{S}$. Disregarding the actual different storage technology, without lack of generalization, we can characterize these devices by charging efficiency, discharging efficiency, leakage rate, maximum capacity, and maximum charging rate, as modeled in the sequel. Hence, for each user $i \in \mathcal{S}$, it is useful to define: the charging and discharging inefficiencies $0<\beta_{i}^{(+)} \leq 1$ and $\beta_{i}^{(-)} \geq 1$, the leakage rate $0<\alpha_{i} \leq 1$, the maximum capacity $\zeta_{i}$, and the maximum hourly charging rate $s_{i}^{\max }$. The current charge level in the device equals the previous slot charge level decreased by the leakage rate and corrected by the energy storage profile. However, the latter is altered through the charging and discharging inefficiency. As in [7], we define it as:

$$
q_{i}(h)=\alpha_{i} q_{i}(h-1)+\beta_{i}^{\top} s_{i}(h), \quad \forall i \in \mathcal{S}
$$

where the vector $s_{i}(h)=\left[s_{i}^{(+)}(h), s_{i}^{(-)}(h)\right]^{\top}$ collects the battery charging and discharging profile at time slot $h$, and $\beta_{i}=\left[\beta_{i}^{(+)},-\beta_{i}^{(-)}\right]^{\top}$ collects the charging and discharging inefficiencies. While in [7] and [9] the authors perform an isolated daily optimization process and consider a constraint on the charge level for the last time slot of one day, here we employ a rolling-horizon approach and introduce a minimum charge level $q_{i}^{\min }$ for each time slot, designed to prevent damages on the devices:

$$
q_{i}^{\min }-\alpha_{i} q_{i}(h-1) \leq \beta_{i}^{\top} s_{i}(h) \leq \zeta_{i}-\alpha_{i} q_{i}(h-1), \quad \forall i \in \mathcal{S} .
$$

Furthermore, the maximum charging and discharging rate must be respected [21], i.e.,

$$
-s_{i}^{\max } \leq \beta_{i}^{\top} s_{i}(h) \leq s_{i}^{\max }, \quad \forall h \in \mathcal{H}, \forall i \in \mathcal{S} .
$$

As for the distributed generation, at each time slot $h$ we have that $s_{i}^{(+)}(h)=s_{i}^{(-)}(h)=0$ for all the prosumers $i \notin \mathcal{S}$. Moreover, it is possible to define for each producer $i \in \mathcal{S}$ an energy storage scheduling vector $\boldsymbol{s}_{i}=\left(\left(s_{i}^{(+)}(h)\right)_{h \in \mathcal{H}},\left(s_{i}^{(-)}(h)\right)_{h \in \mathcal{H}}\right)$ and a set of feasible strategies:

$$
\boldsymbol{\Omega}_{\boldsymbol{s i}}=\left\{\boldsymbol{s}_{i} \in \mathbb{R}_{\geq 0}^{2 H} \mid(9),(10),(11) \text { hold }\right\}, \quad \forall i \in \mathcal{S} .
$$




\section{Energy cost and pricing market}

Let us now denote by $\omega(h)$ the power generated at time slot $h$ by the wind turbine connected with the grid and $L(h)$ the total aggregate grid load for time slot $h$, with:

$$
L(h)=\sum_{i \in \mathcal{D}} l_{i}(h), \quad \forall h \in \mathcal{H} .
$$

As in [22], we consider a function $C_{h}$ to model the cost per unit of energy bought from the distribution system as:

$$
C_{h}(L(h), \omega(h))=K_{h}(L(h)-\omega(h)), \quad \forall h \in \mathcal{H}
$$

where $K_{h}>0$ is the fixed price coefficient at the $h$-th slot (so that price results are time-varying). According to (14), the generic user $i \in \mathcal{D}$ per every time slot $h$ pays to the distribution system the amount $K_{h}(L(h)-\omega(h)) l_{i}(h)$ to receive $l_{i}(h)$. Furthermore, as in [9], we include a global constraint on the aggregate per-slot energy load:

$$
L_{\min } \leq L(h) \leq L_{\max }, \quad \forall h \in \mathcal{H}
$$

where we define $L_{\max }$ as the maximum aggregate load that the grid can afford before a blackout occurs and $L_{\min }$ as a lower bound to prevent the turning off of some primary power plants.

We suppose that the central unit defines the global grid constraints taking into account its energy production facilities. Hence, we should include the availability of wind power; however, for the sake of simplicity, we assume that the wind power availability influences only the energy price and is not affecting the limitations on the aggregate load. Lastly, it is important to remark that (15) is a constraint that couples together all the prosumers' decisions.

In the following section we characterize the wind power availability.

\section{WIND POWER CHARACTERIZATION}

\section{A. Wind speed and wind power generation}

Wind power is one of the most important renewable energy sources, and it has been widely developed in recent years. The high fluctuations in the wind power output may lead to a mismatch between power production and electricity demand. The traditional approaches to cope with this issue rely on the hypothesis that the wind power forecasting error follows a normal distribution. However, this assumption neglects the nonlinear relation between wind speed and the generated power. Here, we consider the availability of wind power for the smart grid, under uncertainty on the wind speed, assuming that the relation between the generated power and the wind speed is nonlinear [23]. More precisely, the relation between the wind speed value $v$ and the output power $\omega(v)$ in a conventional wind turbine is a function of several factors. In the turbine, if the wind speed is lower than a cut-in wind speed value $v_{\text {in }}$, the wind cannot defeat the mechanical friction in the system. After this threshold, the output power increases rapidly with the wind speed, following the Betz law until a rated wind speed value $v_{\text {rated }}$ [24]. The turbines are equipped with a braking system, that after this value of rated wind speed, keeps the output equal to the turbine rated power $\omega_{\text {rated }}$. Once the wind speed exceeds a safety cut-off value $v_{\text {out }}$, the turbine stops

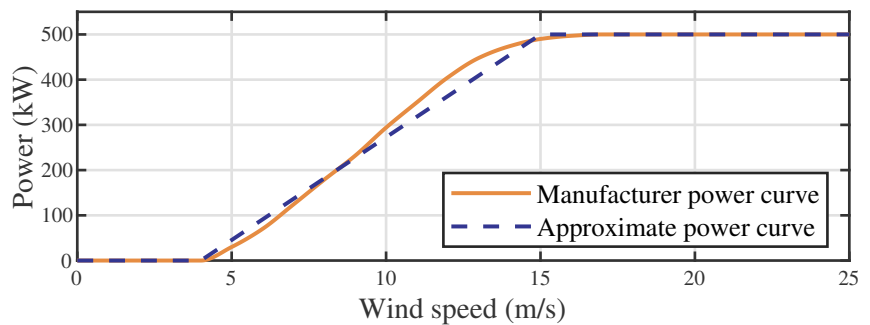

Fig. 2. Manufacturer and approximate power curve for the NedWind-40 wind turbine [26]. The turbine power curve has characteristic parameters: $v_{\text {in }}=4 \mathrm{~m} / \mathrm{s}, v_{\text {rated }}=15 \mathrm{~m} / \mathrm{s}, v_{\text {out }}=25 \mathrm{~m} / \mathrm{s}$ and $\omega_{\text {rated }}=500 \mathrm{~kW}$.

producing energy and is secured by completely stopping the rotor. A reliable yet simple piece-wise linear approximation for this relationship is the following [16]:

$$
\omega(v)=\left\{\begin{array}{lll}
\omega_{\text {rated }} \frac{v-v_{\text {in }}}{v_{\text {rated }}-v_{\text {in }}} & \text { if } & v \in\left[v_{\text {in }}, v_{\text {rated }}\right] \\
0 & \text { if } & v \in\left[0, v_{\text {in }}\right] \cup\left[v_{\text {out }}, \infty\right) \\
\omega_{\text {rated }} & \text { if } & v \in\left[v_{\text {rated }}, v_{\text {out }}\right] .
\end{array}\right.
$$

In Fig. 2 we show the reliability of the proposed approximate power curve (16), by comparing the power curve provided by the manufacturer and the proposed approximate power curve for a typical wind turbine. By using in (16) the expected value $\bar{v}(h)$ obtained by the central unit, it is possible to calculate the wind power production at the expected wind speed value $\bar{\omega}(h)=\omega(\bar{v}(h))$. As an alternative to the expected value approach described overhead, we can describe the behavior of the wind speed $v$ with a PDF that reflects the expectation of the wind speed over a period of time and guides the wind power availability assessment. A PDF can adequately describe the wind speed, based on historical data or wind speed forecasts; the PDF can be either a single function or a combination of two or more functions. The Weibull distribution, Rayleigh distribution, and normal distribution are widely used distributions for modeling the wind speed. We refer to [25], for an extensive review of the PDFs used for wind speed assessment.

\section{B. Historical distribution}

The Weibull and Rayleigh distribution is believed to best describe the wind speed variations over a long term period. Therefore, we assume that the wind speed in a given location follows the Weibull distribution [27], with the $\lambda_{w}$ scale and $k_{w}$ shape parameters. Specifically, we assume that these parameters vary with the turbine location and the period in the year (e.g., for every month). By naming $v$ the wind speed value, we may express, for some given scale and shape parameters, the Weibull wind speed PDF as:

$$
f_{W}(v)= \begin{cases}\frac{k_{w}}{\lambda_{w}}\left(\frac{v}{\lambda_{w}}\right)^{k_{w}-1} \exp \left(-\left(\frac{v}{\lambda_{w}}\right)\right)^{k_{w}} & \text { if } \quad v \geq 0 \\ 0 & \text { if } \quad v<0\end{cases}
$$

Figure 3 shows some examples of the Weibull distribution with different $\lambda_{w}$ and $k_{w}$ parameters. A high value of $k_{w}$, e.g. 3.5, indicates that the difference between the hourly mean wind speed (MWS) and the annual MWS is little. Viceversa, a low value of $k_{w}$, e.g. 2 , means a high divergence from the 
(a)

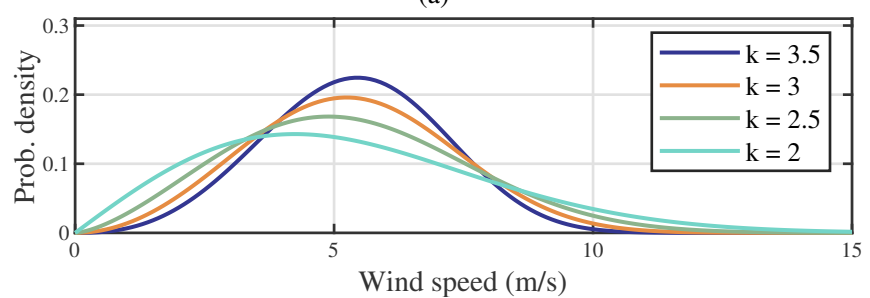

(b)

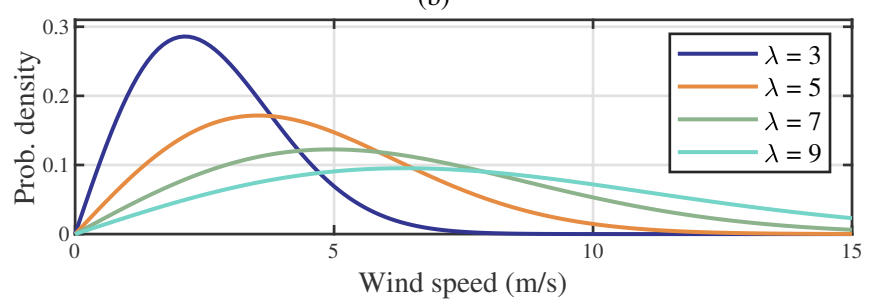

Fig. 3. Weibull distributions with: (a) $\lambda_{w}=6$ and different $k_{w}$ values; (b) $k_{w}=2$ and different $\lambda_{w}$ values.

annual MWS. The value of $\lambda_{w}$ reflects the average wind speed of the wind farm. When a location has a high value of $\lambda_{w}$, it has a high average wind speed; however, this indicates also a significant variation from the annual MWS.

We now characterize the wind power PDF $f(\omega)$ by modifying the approach presented in [17]. Due to the turbine performance curve, the probability that the output power $\omega$ is null can be calculated considering the cumulative probability $F_{v}\left(v_{\text {in }}\right)$ that $v<v_{\text {in }}$ and the cumulative probability $F_{v}\left(v_{\text {out }}\right)$ that $v>v_{\text {out }}$, and is therefore:

$$
f(0)=\mathbb{P}[\omega=0]=F_{v}\left(v_{\text {in }}\right)+\left(1-F_{v}\left(v_{\text {out }}\right)\right) .
$$

Moreover, the probability that $\omega=\omega_{\text {rated }}$ can be calculated considering the cumulative probability that $v \in\left[v_{\text {rated }}, v_{\text {out }}\right]$, and is:

$$
f\left(\omega_{\text {rated }}\right)=\mathbb{P}\left[\omega=\omega_{\text {rated }}\right]=F_{v}\left(v_{\text {out }}\right)-F_{v}\left(v_{\text {rated }}\right) .
$$

In the interval $\left[0, \omega_{\text {rated }}\right]$, the PDF of the generated power $\omega$ can be obtained via a one-to-one mapping with the wind speed [28]. By denoting $A=\left(\frac{v_{\text {rated }}}{v_{\text {in }}}\right)-1$ and $C=\frac{v_{\text {in }}}{\omega_{\text {rated }}}$, we have that:

$$
\left.f(\omega)=\frac{k_{w} A C}{\lambda_{w}}\left(\frac{\left(1+\frac{A \omega}{\omega_{\text {ratece }}}\right)}{\lambda_{w}}\right)^{\text {in }}\right)^{k_{w}-1} \exp \left(-\left(\frac{\left(1+\frac{A \omega}{\omega_{\text {rated }}}\right) v_{\text {in }}}{\lambda_{w}}\right)^{k_{w}}\right) .
$$

Summing up, given the turbine parameters, the location, and period in the year, we can define the historical PDF for the generated wind power as in (21).

The scale $\lambda_{w}$ and the shape $k_{w}$ parameters of the Weibull distribution have a significant impact on the wind power historical PDF. Indeed, for a particular turbine, a different period of the year leads to different Weibull parameters $\lambda_{w}$ and $k_{w}$, and consequently, to a different power PDF. In Fig. 4 we show two examples of the historical PDF with different values for the Weibull parameters. (a)

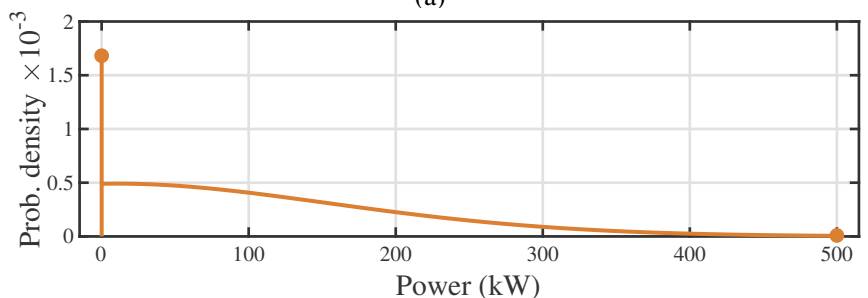

(b)

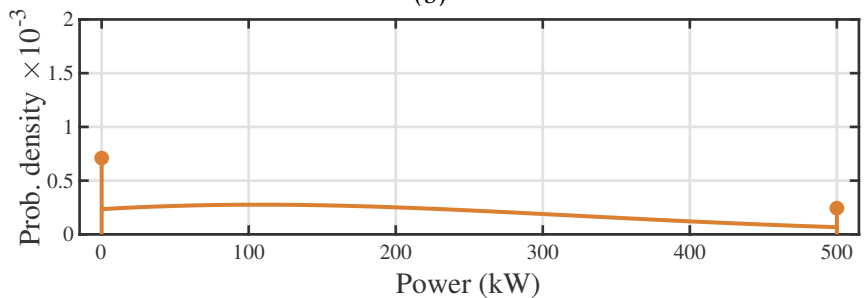

Fig. 4. Wind power historical PDF (21) for different Weibull parameters, calculated for the NedWind-40, with $v_{\text {in }}=4 \mathrm{~m} / \mathrm{s}, v_{\text {rated }}=15 \mathrm{~m} / \mathrm{s}, v_{\text {out }}=25 \mathrm{~m} / \mathrm{s}$ and $\omega_{\text {rated }}=500 \mathrm{~kW}$. (a) $\lambda_{w}=6$ and $k_{w}=2$. (b) $\lambda_{w}=9$ and $k_{w}=2$.

\section{Forecast distribution}

The previous methodology to define a PDF for wind power is based on historical data, and therefore describes the uncertainty of wind speed in the long term. However, it neglects the availability of updatable wind speed forecasts, i.e., a series of forecasts which may be updated at each time shift of the predictive control horizon. Conversely, in this paper, we assume to have a series of wind speed forecasts for the whole control horizon and that the forecasting error is a random variable following a normal distribution [17]. In detail, we assume that to every forecasted wind speed value $\mu$ it is possible to assign a standard deviation $\sigma$ based on the prediction reliability.

By following the aforementioned framework to compute the historical PDF, we calculate the probability that $\omega=0$ by considering the cumulative probability that $v<v_{\text {in }}$ and that $v>v_{\text {out }}$, as in (18). Moreover, the probability that $\omega=\omega_{\text {rated }}$ can be calculated as in (19), considering the cumulative probability that $v \in\left[v_{\text {rated }}, v_{\text {out }}\right]$.

In the interval $\left[0, \omega_{\text {rated }}\right]$, the PDF of the generated power, by denoting again $A=\left(\frac{v_{\text {rated }}}{v_{\text {in }}}\right)-1$ and $C=\frac{v_{\text {in }}}{\omega_{\text {rated }}}$, is:

$$
f(\omega)=\frac{C}{\sqrt{2 \pi \sigma^{2}}} \exp \left(-\frac{\left(\left(1+\frac{A \omega}{\omega_{\text {rated }}}\right) v_{\text {in }}-\mu\right)^{2}}{2 \sigma^{2}}\right)
$$

Summarizing, let us define a forecast PDF for the generated power, given a forecasted wind speed value $\mu$ and a standard deviation $\sigma$, in (24).

Figure 5 shows that the wind power forecast PDF cannot be expressed by the normal distribution due to the nonlinear relation between wind speed and wind power. Indeed, the wind power forecast PDF has an asymmetric distribution, with different peak values.

The range for the wind power PDF varies from 0 to the rated power, consisting of a continuous part and two of impulse functions. When the wind speed is lower than the cut-in wind speed or higher than the cut-out wind speed, the PDF of the 


$$
\begin{aligned}
& \text { Historical PDF: } f(\omega)= \begin{cases}\left(1-\exp \left(-\left(\frac{v_{\text {in }}}{\lambda_{w}}\right)^{k_{w}}\right)+\exp \left(-\left(\frac{v_{\text {out }}}{\lambda_{w}}\right)^{k_{w}}\right)\right) \delta(\omega) & \text { if } \quad \omega=0 \\
\frac{k_{w} A C}{\lambda_{w}}\left(\frac{\left(1+\frac{A \omega}{\omega_{\text {rated }}}\right) v_{\text {in }}}{\lambda_{w}}\right)^{k_{w}-1} \exp \left(-\left(\frac{\left(1+\frac{A \omega}{\omega_{\text {rated }}}\right) v_{\text {in }}}{\lambda_{w}}\right)\right. & \text { if } \quad \omega \in\left(0, \omega_{\text {rated }}\right)\end{cases} \\
& \left(\exp \left(-\left(\frac{v_{\text {rated }}}{\lambda_{w}}\right)^{k_{w}}\right)-\exp \left(-\left(\frac{v_{\text {out }}}{\lambda_{w}}\right)^{k_{w}}\right)\right) \delta(\omega) \quad \text { if } \omega=\omega_{\text {rated }} \\
& \text { ( } 0 \text { if } \omega>\omega_{\text {rated }} \\
& \text { Forecast PDF: } \quad f(\omega)= \begin{cases}\left(\frac{1}{\sqrt{2 \pi \sigma^{2}}}\left(\int_{-\infty}^{v_{\text {in }}} \exp \left(-\frac{(v-\mu)^{2}}{2 \sigma^{2}}\right) d v+\int_{v_{\text {out }}}^{+\infty} \exp \left(-\frac{(v-\mu)^{2}}{2 \sigma^{2}}\right) d v\right)\right) \delta(\omega) & \text { if } \quad \omega=0 \\
\frac{C}{\sqrt{2 \pi \sigma^{2}}} \exp \left(-\frac{\left(\left(1+\frac{A \omega}{\omega_{\mathrm{r}}}\right) v_{\text {in }}-\mu\right)^{2}}{2 \sigma^{2}}\right) & \text { if } \omega \in\left(0, \omega_{\text {rated }}\right) \\
\left(\frac{1}{\sqrt{2 \pi \sigma^{2}}} \int_{v_{\mathrm{r}}}^{v_{\text {out }}} \exp \left(-\frac{(v-\mu)^{2}}{2 \sigma^{2}}\right) d v\right) \delta(\omega) & \text { if } \quad \omega=\omega_{\text {rated }} \\
0 & \text { if } \omega>\omega_{\text {rated }}\end{cases}
\end{aligned}
$$

have more reliable forecasts.

Reference [29] shows that uncertainty is strongly related to the wind speed value and the forecasting horizon. These relations, together with the uncertainty related to a specific weather prediction model and the wind farm location and geography, can be estimated using historical data.

\section{Instability index}

The weather conditions are not always the same; a more stable atmospheric situation empowers better forecasts, reducing uncertainty in the wind power forecast. To evaluate the weather condition, [30] introduces a methodology to assess the risk of short-term wind power forecasts by using a meteorological risk index. The index relies on the ensemble forecasting methodology. Instead of making a single prediction of the most likely weather, a set of forecasts is produced. The set aims at indicating the range of possible future states of the atmosphere. This is usually made by changing the initial condition of the model and observing how the results are perturbed. Differently from [30], we define an instability index obtained by evaluating the error made by forecasts of different time instances.

In detail, let us evaluate the stability of the weather conditions at the generic time slot $k$. Hence, we denote with $v_{r}$ the registered wind speed at the previous time slot $k-1$, and $v_{t}$ the forecasted wind speed value for time slot $k-1$ obtained at time slot $(k-1)-t$, i.e., the forecast made at the time slot $(k-1)-t$ for the time slot $k-1$. By collecting the previously recorded and forecasted data, each user calculates

- the weather "stability", i.e., stable weather conditions 
(a)

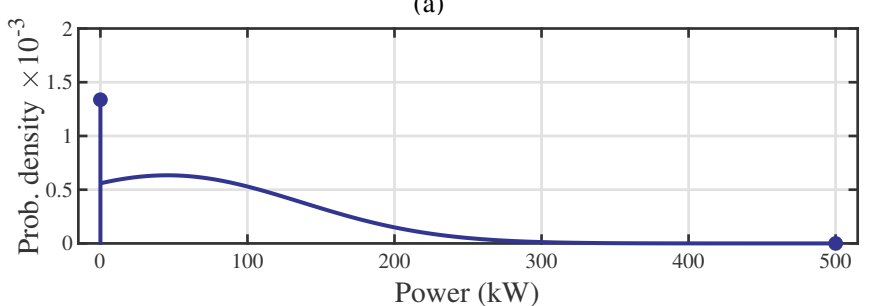

(b)

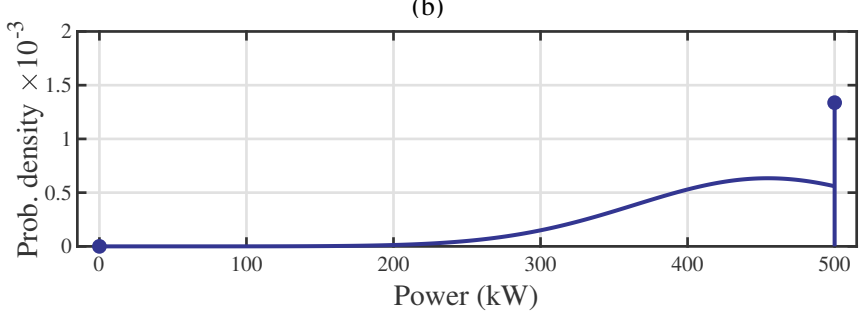

(c)

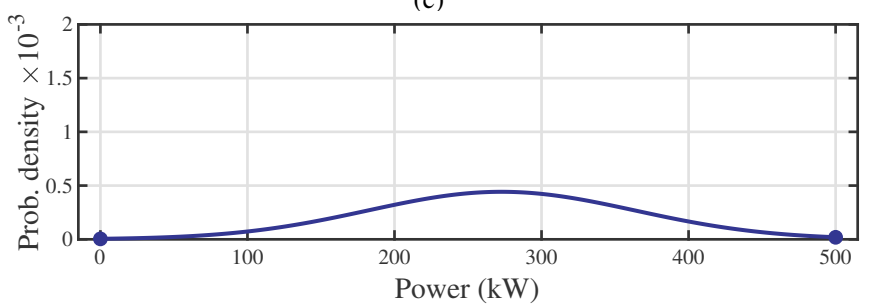

Fig. 5. Wind power forecast PDF (24) for different wind speed values. Calculated for the NedWind-40, with $v_{\text {in }}=4 \mathrm{~m} / \mathrm{s}, v_{\text {rated }}=15 \mathrm{~m} / \mathrm{s}, v_{\text {out }}=$ $25 \mathrm{~m} / \mathrm{s}$ and $\omega_{\text {rated }}=500 \mathrm{~kW}$. (a) $\mu=5 \mathrm{~m} / \mathrm{s}$ and $\sigma=2 \mathrm{~m} / \mathrm{s}$. (b) $\mu=14 \mathrm{~m} / \mathrm{s}$ and $\sigma=2 \mathrm{~m} / \mathrm{s}$. (c) $\mu=10 \mathrm{~m} / \mathrm{s}$ and $\sigma=2 \mathrm{~m} / \mathrm{s}$.

the instability index as:

$$
n=\sum_{t=1}^{T} \tau_{t}\left|v_{r}-v_{t}\right|
$$

where $T$ is the number of previous forecasts employed to calculate the index, and $\tau_{t}$ are weights that give less importance to the error made by the older predictions, i.e., the error $\left|v_{r}-v_{1}\right|$ should has a stronger impact than $\left|v_{r}-v_{T}\right|$ in evaluating the index. Moreover, we assume that $\sum_{t=1}^{T} \tau_{t}=1$.

As we show in the numerical experiments, the forecasting error increases linearly with the instability index. At every time slot $k$, we calculate the instability index employing the data related to the previous time slot $k-1$, and we modify the standard deviation for the subsequent forecasts. For instance, when the index is low, we assume that the forecast is accurate, and therefore, we reduce the standard deviation of the next time slots' forecasts. However, as the weather conditions typically change within a few hours, we do not employ the instability index to modify the standard deviation value for the forecast of the entire control horizon $H$.

\section{E. Composed distribution}

Highly uncertain forecasts are caused by high wind speed values, long prediction horizon, or unstable weather conditions. In some cases, uncertainty can be considerably high, leading to an overestimation in the forecast PDF. A combination of the information contained in the historical and the forecast PDFs can reduce this problem. The authors in [31] (a)

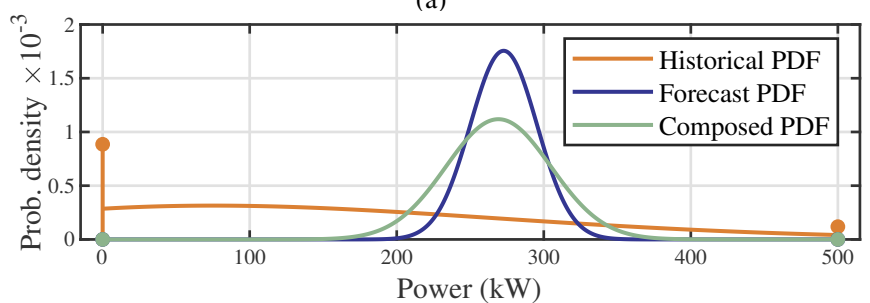

(b)

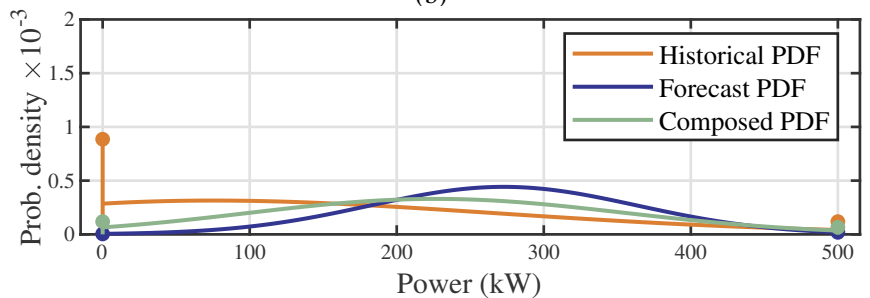

Fig. 6. Composed wind power PDF (23) with different standard deviation values. Calculated for the NedWind-40, with $v_{\text {in }}=4 \mathrm{~m} / \mathrm{s}, v_{\text {rated }}=15 \mathrm{~m} / \mathrm{s}$, $v_{\text {out }}=25 \mathrm{~m} / \mathrm{s}, \omega_{\text {rated }}=500 \mathrm{~kW}, \mu=10 \mathrm{~m} / \mathrm{s}, \lambda_{w}=8$ and $k_{w}=2$. (a) $\sigma=0.5 \mathrm{~m} / \mathrm{s}$. (b) $\sigma=2 \mathrm{~m} / \mathrm{s}$.

present several ways to combine PDFs. In particular, let us employ the logarithmic method:

$$
f(\omega)=c f_{1}(\omega)^{w_{1}} f_{2}(\omega)^{w_{2}}
$$

where $f_{1}(\omega)$ is the historical PDF, $f_{2}(\omega)$ the forecast PDF, $w_{1}$ and $w_{2}$ are weights with $w_{1}+w_{2}=1$, and $c$ a normalizing constant. It is worth pointing out that the weight indicates the PDF's influence. For the sake of simplicity, in the rest of the paper, we employ the same weight to all the PDFs, i.e., $w_{1}=$ $w_{2}=0.5$. In Fig. 6 we show an example of this methodology. In the first figure, the forecast PDF has a smaller standard deviation value, which indicates a more accurate and reliable prevision. The composed PDF, in this case, is comparable to the forecast PDF. On the other hand, in the second figure, the forecast has high uncertainty, and the composed PDF is corrected by the information contained in the historical PDF.

\section{The Distributed Stochastic CONTROL Algorithm For DEMAND Side MANAGEMENT}

The objective of this section is to define the overall optimization problem for the smart grid model described in Section II.

\section{A. Game model}

Game theory describes and analyzes scenarios with interactive decisions. Noncooperative game theory is a branch of game theory employed to solve conflicts between interacting players [32]. Considering our smart grid model, and bearing in mind the users' selfish behavior and the aforementioned cost function, for a generic user the price of energy depends on the other users' strategy. Hence, it is meaningful to model the optimization problem as a noncooperative game, as proposed in [7].

In the noncooperative game, each player behaves selfishly to optimize its own welfare, generally quantified through a 
cost function. A noncooperative game is usually defined by its three components:

- the players, that in our case are all the prosumers $i \in \mathcal{N}$ participating in the grid optimization process;

- a cost function $J_{i}$ for each player;

- the strategy of each player $\boldsymbol{x}_{i}=\left(\boldsymbol{d}_{i}, \boldsymbol{g}_{i}, \boldsymbol{s}_{i}\right)^{\top}$, which corresponds to a specific consumption, generation and storage profile.

In the model, according to (14), the energy price at each time slot is a function of the aggregate load and the wind power generation; the latter is here regarded as a stochastic variable. Accordingly, for each active user $i \in \mathcal{N}$ we can define the cost function as the monetary exchange with the distribution system over the control horizon $\mathcal{H}$. Let us define the strategy vector for the generic prosumer $i \in \mathcal{N}$ at the generic time slot $h$ as $x_{i}(h)=\left(d_{i}(h), g_{i}(h), s_{i}(h)\right)^{\top}$, the strategy scheduling vector $\boldsymbol{x}_{i}=\left(\boldsymbol{d}_{i}, \boldsymbol{g}_{i}, \boldsymbol{s}_{i}\right)^{\top}$ and the local feasible strategy set that takes into account the individual user preferences as:

$\Omega_{\boldsymbol{x}_{i}}=\left\{\boldsymbol{x}_{i} \in \mathbb{R}^{4 H} \mid \boldsymbol{d}_{i} \in \Omega_{\boldsymbol{d}_{i}}, \boldsymbol{g}_{i} \in \Omega_{\boldsymbol{g}_{i}}, \boldsymbol{s}_{i} \in \Omega_{\boldsymbol{s}_{i}}\right\}, \forall i \in \mathcal{N}$.

Then, by using the pricing model in (14) and by naming with $\boldsymbol{x}_{-i}$ the collection of the strategy scheduling vectors $\boldsymbol{x}_{j}$ of all users $j \in \mathcal{D} \backslash\{i\}$, the total expense for a generic prosumer $i \in \mathcal{N}$ over the control horizon $\mathcal{H}$ is:

$$
\begin{gathered}
J_{i}\left(\boldsymbol{x}_{i}, \boldsymbol{x}_{-i}, \boldsymbol{\omega}\right)=\sum_{h \in \mathcal{H}} K_{h}\left(l_{-i}(h)+e_{i}(h)+\delta^{\top} x_{i}(h)-\omega(h)\right) . \\
\cdot\left(e_{i}(h)+\delta^{\top} x_{i}(h)\right)+\sum_{h \in \mathcal{H}} W_{i}\left(\delta_{g}^{T} x_{i}(h)\right)
\end{gathered}
$$

where $l_{-i}(h)=\sum_{i \in \mathcal{D} \backslash\{i\}} l_{i}(h)$ is the aggregate per-slot load of all the players $j \in \mathcal{D} \backslash\{i\}$, while $\delta=(1,-1,1,-1)^{\top}$ and $\delta_{g}=(0,1,0,0)^{\top}$ are auxiliary vectors. In our setting for the generic time slot $h$, the available wind power $\omega(h)$ is a stochastic variable that reveals its value ex-post. Therefore, users cannot calculate the exact value of $J_{i}$ in advance.

Although each user acts selfishly, by choosing a strategy included in its local feasible set $\Omega_{\boldsymbol{x}_{i}}$, the global grid constraint in (15) must be respected. Hence, we should consider a noncooperative generalized game, where the coupling between the players occurs not only via the cost functions but additionally through a collective global feasible set. In our settings, the coupling constraints are introduced by an affine function, $\boldsymbol{x} \mapsto A \boldsymbol{x}-b$, where $A \in \mathbb{R}^{2 H \times 4 H N}$ and $b \in \mathbb{R}^{2 H}$. In particular, let us define $b=\left[L_{\max }-L_{P}(k), \ldots, L_{\max }-L_{P}(k+\right.$ $\left.H-1), L_{P}(k)-L_{\min }, \ldots, L_{P}(k+H-1)-L_{\min }\right]^{\top}$, with $L_{P}(h)=\sum_{i \in \mathcal{P}} l_{i}(h)$ the cumulative consumption of the passive user $i \in \mathcal{P}$, and $A=\left[A_{1}, \ldots, A_{N}\right]=\mathbf{1}_{N} \otimes \Delta$, being $\Delta=\left[I_{H},-I_{H}, I_{H},-I_{H}\right], I_{H} \in \mathbb{R}^{H \times H}$ the identity matrix and $\otimes$ the Kronecker product. We remark that $A_{i}$ outlines how the active user $i \in \mathcal{N}$ is involved in the global coupling constraints.

Thus we can now define the collective global feasible set $\mathcal{X}$ as the intersection between the collection of the local feasible sets of all the prosumers $\Omega=\prod_{i=1}^{N} \Omega_{\boldsymbol{x}_{i}}$ and the coupling constraint. More formally, we have, therefore:

$$
\mathcal{X}=\boldsymbol{\Omega} \cap\left\{\boldsymbol{x} \in \mathbb{R}^{4 H N} \mid A \boldsymbol{x}-b \leq \mathbf{0}_{2 H}\right\} .
$$

where $\boldsymbol{x}=\left(\boldsymbol{x}_{i}\right)_{i=1}^{N}$ is the collective strategy scheduling vector.

Overall, we obtain $N$ inter-dependent optimization problems as in the following:

$$
\forall i \in \mathcal{N}:\left\{\begin{array}{c}
\underset{x_{i} \in \mathbb{R}^{4 H}}{\operatorname{argmin}} \tilde{J}_{i}\left(\boldsymbol{x}_{i}, \boldsymbol{x}_{-i}, \boldsymbol{\omega}\right) \\
\text { s.t. }\left(\boldsymbol{x}_{i}, \boldsymbol{x}_{-i}\right) \in \mathcal{X}
\end{array}\right.
$$

where $\tilde{J}_{i}$, is the assumption that each active user $i \in \mathcal{N}$ makes for the real cost function in (28), given the wind speed forecast provided by the central unit. We remark that (28) is a function of a stochastic variable, therefore, each user tries to define a function that reflects it as much as possible.

The formulation in (30) defines a generalized Nash equilibrium (GNE) problem that we can indicate in compact form as $\mathscr{G}=(\mathcal{X}, \tilde{\mathbf{J}})$, where $\mathcal{X}$ is the collective global feasible set as in (29) and $\tilde{\mathbf{J}}=\left(\tilde{J}_{i}\left(\boldsymbol{x}_{i}, \boldsymbol{x}_{-i}, \boldsymbol{\omega}\right)\right)_{i=1}^{|\mathcal{N}|}$ is the collection of the active users' cost function. In the game theory solving the GNE problem in (30) means the computation of a GNE, which is a collective strategy profile $\boldsymbol{x}^{*} \in \mathcal{X}$ with the property that no single player can benefit from a unilateral deviation from $\boldsymbol{x}_{i}^{*}$, if all the other players act according [32]. More formally, we have:

$$
J_{i}\left(\boldsymbol{x}_{i}^{*}, \boldsymbol{x}_{-i}^{*}\right) \leq \inf \left\{J_{i}\left(\boldsymbol{x}_{i}, \boldsymbol{x}_{-i}^{*}\right) \mid\left(\boldsymbol{x}_{i}, \boldsymbol{x}_{-i}^{*}\right) \in \mathcal{X}\right\} .
$$

In the rest of this Section, we introduce an algorithm to compute a GNE in a semi-decentralized fashion. Moreover, we present two variants of the cost function $\tilde{J}_{i}$, i.e., the guess made by the $i$ th user given the stochasticity of the wind power availability.

\section{B. Semi-decentralized equilibrium computation}

Once we have defined the overall aggregated game $\mathscr{G}=$ $(\mathcal{X}, \tilde{\mathbf{J}})$, let us now employ the preconditioned forward backward $(\mathrm{pFB})$ algorithm presented in [33] to compute an equilibrium in a semi-decentralized fashion.

In Algorithm 1, at every time slot, the central unit broadcasts an initial value for the so-called incentive signal $\lambda$. At each iteration, every user attempts to reduce his cost function, given the current value for the grid coefficients, the wind forecast, and the aggregative load on the grid, taking a gradient step of length $\gamma$ projected into the feasible local set. Next, the central unit collects all the users' strategies $\boldsymbol{x}$, then it calculates the incentive signal $\lambda^{+}$for the subsequent iteration taking into account the expected constraint violation. Moreover, the central unit broadcasts to each prosumer $i \in \mathcal{N}$ the vector $\boldsymbol{l}_{-i}=\left(\sum_{i \in \mathcal{D} \backslash\{i\}} l_{i}(h)\right)_{h \in \mathcal{H}}$. This is directly related to the strategies of all the other users $\boldsymbol{x}_{-i}$, however, due to the aggregation does not contain any private information. Finally, the central unit terminates the iterative process when an adequate termination criterion is reached, i.e., when all the strategies and the incentive signal $\lambda$ converge. Note that the pFB algorithm guarantees the convergence to the unique variational GNE, therefore, all agents are penalized in the same way in order to satisfy the shared constraints. This approach is economically fair because the cost for fulfilling the common constraints is fairly shared, i.e., all agents are subject to the same dual variables $\left(\lambda_{i}=\lambda_{j}, \forall i, j\right)$ [33]. 


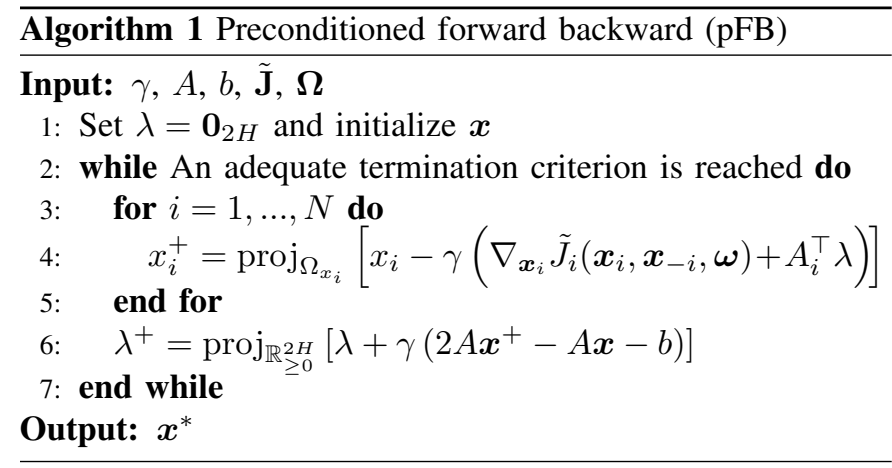

\section{Expected value formulation}

In the proposed pricing scheme, the energy price is a function of an aleatory variable, such as the wind power availability. Moreover, we suppose that active users know the characteristic turbine parameters and that the central unit broadcasts at every time slot the wind speed forecast for the entire control horizon. Therefore, each active user decides how to manage the uncertainty in the wind speed forecasts. The most straightforward way to approach the problem is to consider the wind power availability as a deterministic variable by employing the expected value of the wind speed forecast $\bar{v}(h)$. By using (16), it is then possible to calculate the expected value for the wind power production $\bar{\omega}(h)$. Calling $\overline{\boldsymbol{\omega}}=(\bar{\omega}(h))_{h \in \mathcal{H}}$ the wind power expected production vector, we can now formally define the cost function for a generic active user that employs the expected value approach as:

$$
\begin{gathered}
\bar{J}_{i}\left(\boldsymbol{x}_{i}, \boldsymbol{x}_{-i}, \overline{\boldsymbol{\omega}}\right)=\sum_{h \in \mathcal{H}} K_{h}\left(l_{-i}(h)+e_{i}(h)+\delta^{T} x_{i}(h)-\bar{\omega}(h)\right) . \\
\cdot\left(e_{i}(h)+\delta^{T} x_{i}(h)\right)+\sum_{h \in \mathcal{H}} W_{i}\left(\delta_{g}^{T} x_{i}(h)\right) . \quad \text { (32) }
\end{gathered}
$$

It is worth pointing out that (28) determines how much a generic user actually pays to the grid after the realization of the stochastic variable (the so-called wait-and-see value), while (32) indicates how much the user is presuming to pay by employing this approach, which is different from the expected value of the cost function.

\section{Approximated stochastic programming}

The main issue in employing the expected value approach is that the presence of a stochastic variable may lead to a non-optimal energy schedule; this opens the door to a wealth of different approaches to cope with this problem. Stochastic programming is a framework used to solve models with uncertainty, taking advantage of the fact that the PDF ruling the random variable is known or can be in some way estimated. More specifically, we suppose that the cost function of a generic user is a stochastic variable itself [34], with expected value:

$$
\mathbb{J}_{i}\left(\boldsymbol{x}_{i}, \boldsymbol{x}_{-i}, \boldsymbol{\omega}\right)=\mathbb{E}_{\omega}\left[J_{i}\left(\boldsymbol{x}_{i}, \boldsymbol{x}_{-i}, \boldsymbol{\omega}\right)\right] .
$$

Determining the exact solution of (33) is complicated; an alternative solution methodology replaces the random variable by finite random samples and solves a resulting deterministic optimization problem [35]. This methodology is often called Sample Average Approximation (SAA). This technique is mainly based on having a prior known PDF, from which $M$ independent and identically distributed (IID) random samples of the stochastic variable are obtained. In our case, by using $M$ samples obtained through one of the aforementioned wind power PDFs, (21) or (24) or (26), we can define an approximated cost function for a generic active user $i \in \mathcal{N}$, as:

$$
\hat{J}_{i}\left(\boldsymbol{x}_{i}, \boldsymbol{x}_{-i}, \hat{\boldsymbol{\omega}}_{i}\right)=\frac{1}{M_{i}} \sum_{m=1}^{M_{i}} J_{i}^{m}\left(x_{i}, \boldsymbol{x}_{-i}, \boldsymbol{\omega}_{i}^{m}\right)
$$

where $\boldsymbol{\omega}_{i}^{m}$ is one of the samples for the wind power production vector, $M_{i}$ is the number of samples that the generic user decides to perform, $J_{i}^{m}$ is the cost function defined with the $m$ th samples, and $\hat{\boldsymbol{\omega}}_{i}=\left(\boldsymbol{\omega}_{i}^{m}\right)_{m=1}^{M_{i}}$ is the collection of all the wind power production vector samples. Note that $\boldsymbol{\omega}_{i}^{m}=$ $\left(\omega(h)_{i}^{m}\right)_{h \in \mathcal{H}}$ is one of the sampled scenarios for the whole control horizon; however, it should be noted that each $\omega(h)_{i}^{m}$ is sampled from a time slot specific PDF.

It is well known that under relatively mild conditions, the defined approximate stochastic solution converges to the "true" stochastic one, as the sample size $M$ increases (the interested readers is referred to the discussion in [34] and [35] on this issue which is beyond the scope of this paper).

\section{E. Rolling-horizon DSM algorithm}

Let us now propose a novel procedure to continuously control prosumers by embedding Algorithm 1 into the receding horizon scheme of Algorithm 2. In particular, at each generic time slot $k$, prosumers participate in the optimization process to define the consumption, generation and storage strategies for the next control horizon $\{k, \ldots, k+H-1\}$.

In the initialization phase of each time shift (line 2 of Algorithm 2), the central unit broadcasts the price coefficients $\boldsymbol{K}^{k}=\left(K_{h}\right)_{h=k}^{k+H-1}$ and the wind speed forecasts $\overline{\boldsymbol{v}}^{k}=$ $(\bar{v}(h))_{h=k}^{k+H-1}$ for the entire control horizon.

Now for clarity, let us divide the active users set $\mathcal{N}$ into $\mathcal{N}_{D}$ and $\mathcal{N}_{S}$, where $\mathcal{N}=\mathcal{N}_{D} \cup \mathcal{N}_{S}$ and $\mathcal{N}_{D} \cap \mathcal{N}_{S}=\varnothing$. The first set comprehends the users that rely on the information given by the central unit to calculate the expected value for the wind power forecast for the control horizon $\overline{\boldsymbol{\omega}}^{k}=(\bar{\omega}(h))_{h=k}^{k+H-1}$ (line 14). Conversely, the second set contains the users utilizing the SAA cost function (34): these users believe that the implementation of an approximated stochastic strategy will bring a profitable advantage. Latter users generate a different PDF for each time slot of the whole control horizon (line 7). The PDFs can be based only on the historical data (21), the wind speed forecast (24), or both (26). In the last two cases, users may also apply the instability index during the PDFs creation phase. Consequently, they are obtaining $M$ IID samplings for every time slot of the control horizon from the PDFs (line 8). Therefore, each user defines its cost function (line 10) as:

$$
\tilde{J}_{i}^{k}= \begin{cases}\bar{J}_{i}^{k}\left(\boldsymbol{x}_{i}^{k}, \boldsymbol{x}_{-i}^{k}, \overline{\boldsymbol{\omega}}^{k}\right) & \text { if } i \in \mathcal{N}_{\mathcal{D}} \\ \hat{J}_{i}^{k}\left(\boldsymbol{x}_{i}^{k}, \boldsymbol{x}_{-i}^{k}, \hat{\boldsymbol{\omega}}_{i}^{k}\right) & \text { if } i \in \mathcal{N}_{\mathcal{S}} .\end{cases}
$$




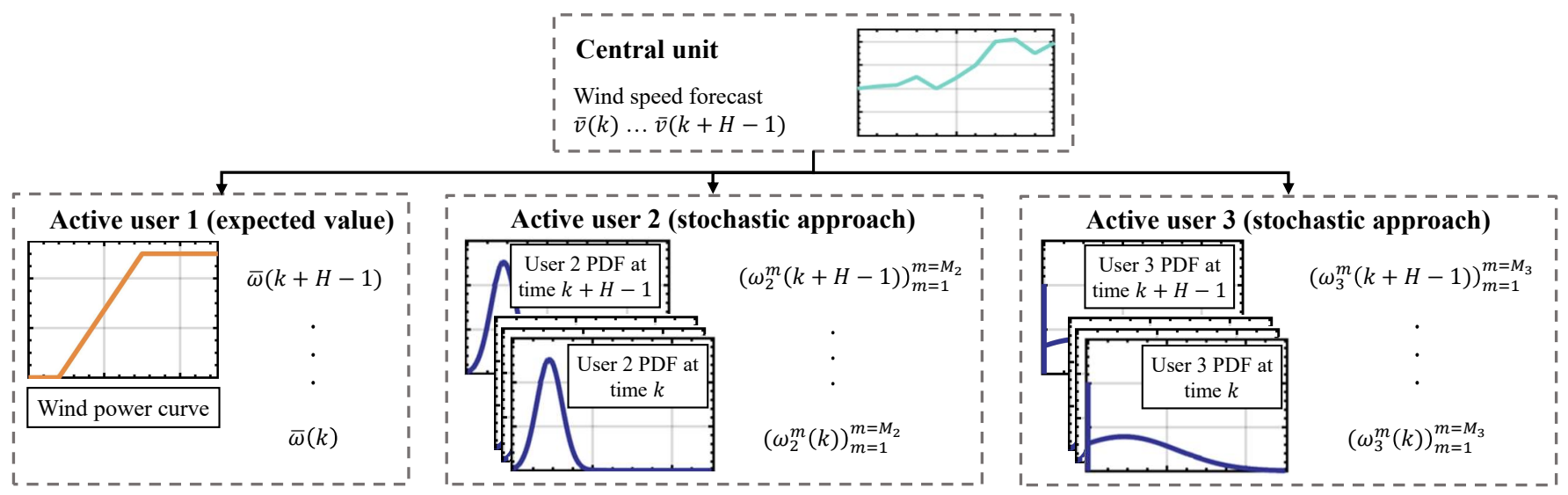

Fig. 7. Illustration of the DSM scheme with three prosumers.

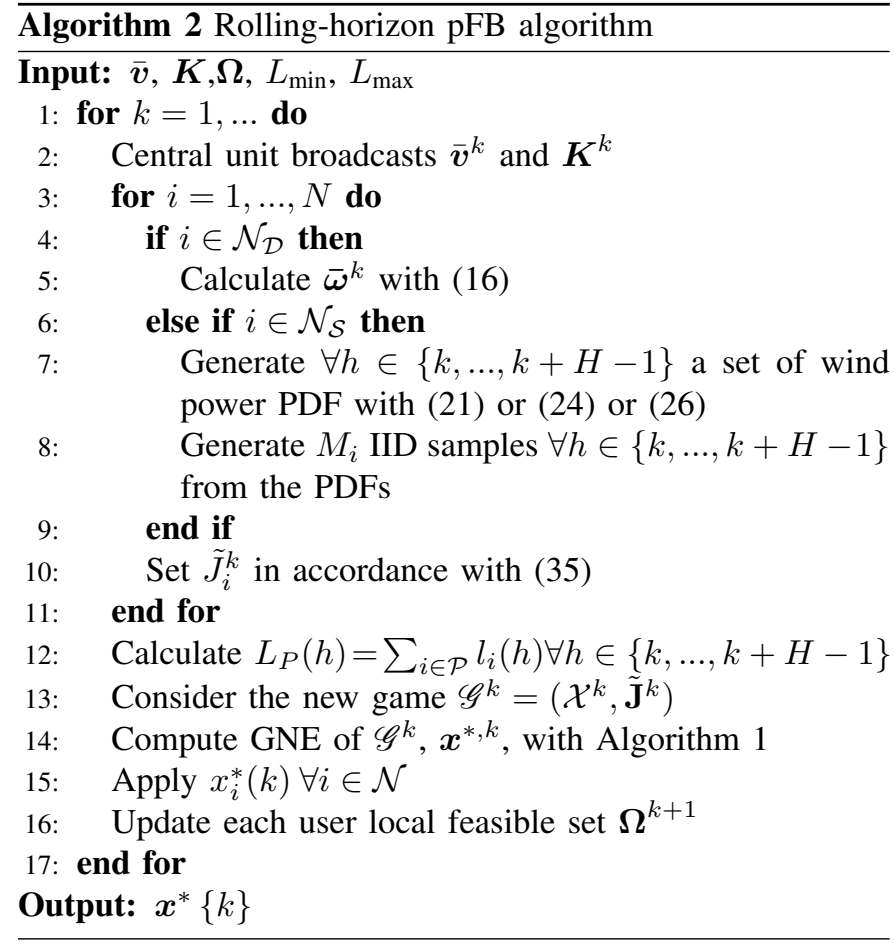

We underline that the superscript $k$ is used to indicate that the cost functions and the variables refer to the control horizon $\{k, \ldots, k+H-1\}$.

A new aggregated game is defined by considering the global feasible set $\mathcal{X}^{k}$ and the cost functions' collection $\tilde{\mathbf{J}}^{k}=$ $\left(\tilde{J}_{i}^{k}\right)_{i=1}^{|\mathcal{N}|}$ (line 12-13). Moreover, the optimization problem is solved for the whole control horizon by calculating an equilibrium via Algorithm 1 (line 14).

The solution of the GNE problem is the optimal strategy $\boldsymbol{x}_{i}^{*, k}$ for each user $i \in \mathcal{N}$. However, following the receding horizon implementation, only the first step of the solution $x_{i}^{*}(k)=\left(g_{i}^{*}(k), s_{i}^{*}(k)\right)^{\top}$ is implemented. Lastly, each user updates its local feasible set $\boldsymbol{\Omega}^{k+1}$ considering the implemented strategy.

\section{Numerical Simulations}

In this section, we present the results of the proposed methodology, obtained using real data from several offshore platforms in the North Sea. Each of these offshore drilling rigs includes a meteorological station that measures the wind speed, and other hourly averaged weather data. In particular, we chose eight platforms connected with the Meteorological Assimilation Data Ingest System (MADIS) [36], from which it is possible to obtain historical data and hourly current measured data. In Table I we show the platforms' coordinates; it is relevant to point out that several existing wind farms are present in the area (e.g., the Orsted Hornsea wind farm

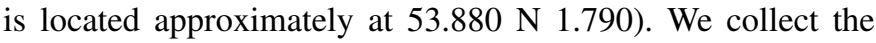
forecasts employing a script that calls every hour within an API several different weather providers to obtain the next 24 hours wind speed forecasts for all the wind farms locations [37] [38]. The employed wind speed forecasts data cover a period of 9 months. We underline that the forecasting models include the data of the offshore platforms through the MADIS.

\section{A. Historical Weibull Parameters}

As discussed earlier, the Weibull parameters have a significant impact on the historical wind power PDF. Therefore, to take into account this issue, we estimate the parameters for each platform and every day of the year. We calculate them by centering a 30-day window on a specific day and by getting all the available historical data for this window. For example, we estimate the Weibull parameters for August 21 by getting all the measured wind speed values in the interval between August 6 and September 5 for all the past available years (e.g., from 2009 to 2019). Table II shows the Weibull parameters calculated employing the data of the entire year and only for the month of May. The period of the year and the location cause the discrepancy between the parameters. For instance, the platforms closer to the coast hold a lower $\lambda_{w}$ than the other platforms.

\section{B. Forecast Standard Deviation}

In our model, we assume that the wind speed forecasts' errors follow a normal distribution. Therefore, the only pa- 
TABLE I

OFFSHORE METEOROLOGICAL STATION LOCATION

\begin{tabular}{ll}
\hline Station code and name & Coordinates \\
\hline Station 62121 - Carrack & $53.500 \mathrm{~N} \mathrm{2.700} \mathrm{E}$ \\
Station 62127 - Cleeton & $54.000 \mathrm{~N} 0.700 \mathrm{E}$ \\
Station 62144 - Clipper & $53.400 \mathrm{~N} 1.700 \mathrm{E}$ \\
Station 62145 - North Sea & $53.102 \mathrm{~N} \mathrm{2.800} \mathrm{E}$ \\
Station 62148 - Barque & $53.600 \mathrm{~N} 1.500 \mathrm{E}$ \\
Station 62149 - West Sole & $53.700 \mathrm{~N} 1.100 \mathrm{E}$ \\
Station 62150 - Amethyst & $53.600 \mathrm{~N} 0.700 \mathrm{E}$ \\
Station 62165 - Ravenspurn N. & $54.000 \mathrm{~N} 1.100 \mathrm{E}$ \\
\hline
\end{tabular}

TABLE II

WEIBULL PARAMETERS FOR THE CONSIDERED STATIONS

\begin{tabular}{lcccc}
\hline Station code & $\lambda_{w}$ (Year) & $k_{w}$ (Year) & $\lambda_{w}$ (May) & $k_{w}$ (May) \\
\hline Station 62121 & 8.92797 & 2.24957 & 7.95649 & 2.61785 \\
Station 62127 & 6.80654 & 1.95317 & 6.81277 & 1.76822 \\
Station 62144 & 8.22549 & 2.09191 & 7.21183 & 2.45309 \\
Station 62145 & 9.33593 & 2.15606 & 8.52988 & 2.48171 \\
Station 62148 & 8.33172 & 2.13873 & 7.48135 & 2.59125 \\
Station 62149 & 8.80511 & 2.17411 & 7.66658 & 2.53036 \\
Station 62150 & 7.94109 & 2.12239 & 6.59681 & 2.38217 \\
Station 62165 & 8.28173 & 2.18134 & 7.17359 & 2.63819 \\
\hline
\end{tabular}

rameter to determine is the standard deviation $\sigma$. We obtain this parameter by performing an analysis of the weather providers' past forecasting errors. More in detail, for each weather provider and each station, we estimate through a linear regression procedure the standard deviation employing the forecasted value and the forecasting horizon. The results are comparable with the literature and show that some weather providers are more accurate than others. Moreover, the relation between the forecasting horizon, the forecasted wind speed value, and the standard deviation can have stronger or weaker importance. We employ the relations mentioned above to assign to each forecast a reasonable standard deviation value. In Fig. 8 we show these relations for one of the meteorological stations mentioned above.

\section{Instability Index}

In this work, we propose an instability index to evaluate the overall weather conditions. We calculate it by comparing the measured wind speed value in a time slot and the six past forecasts made for it. We calculate the relationship between the instability index and the standard deviation empirically. In particular, we bucket the index, and we calculate the standard deviation of the errors of the binned data.

Figure 9(a) shows how the standard deviation changes compared with its mean value. The figure indicates approximately that for values of the index lower than one, the standard deviation is lower than the average value. Furthermore, Fig. 9(b) shows the relative frequency of the instability index. The index is mainly concentrated between 0.5 and 1 ; this indicates quite stable weather conditions, while higher values mean unstable conditions. Lastly, in Fig. 10 we show the instability index variability. The figure shows that the index is highly variable on a long term analysis, while it is relatively stable within a few hours.

At each time slot, we employ the index to increase or reduce the standard deviation of future forecasts. However, due to the

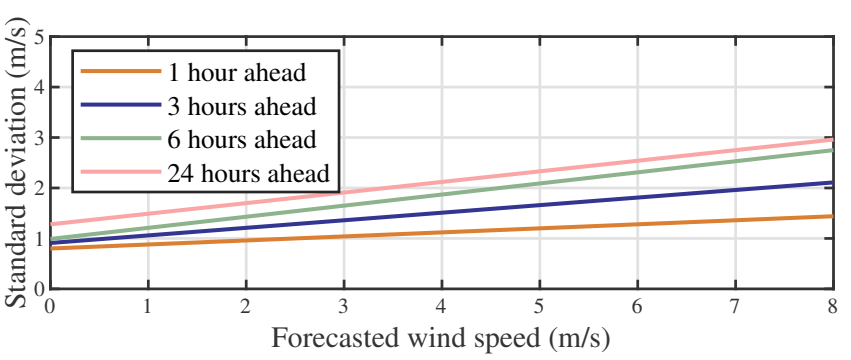

Fig. 8. Correlation between the standard deviation of the prediction error, the predicted wind speed value, and the forecast horizon for the station 62121 .

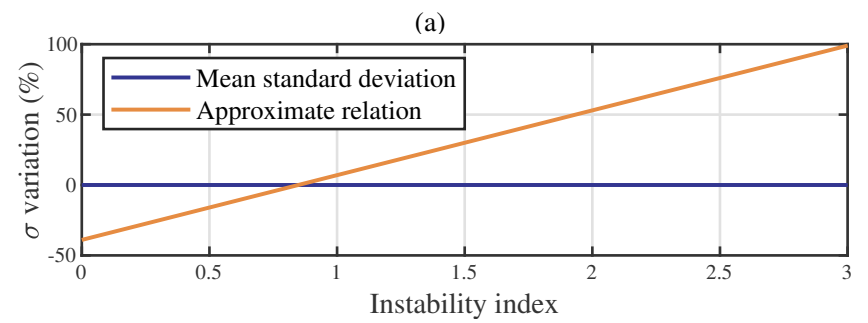

(b)

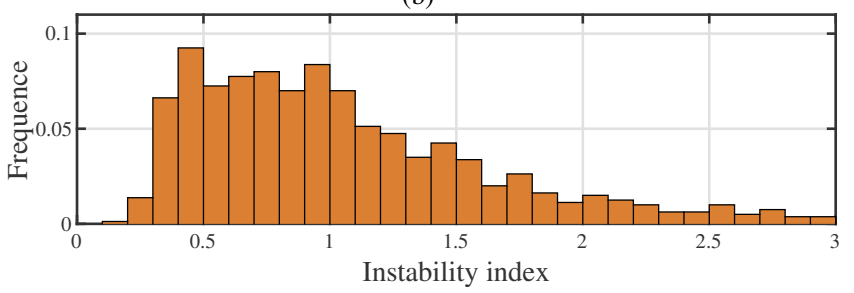

Fig. 9. Instability index for the station 62121: (a) Relative percentage variation of the forecast standard deviation. (b) Relative frequency.

(a)

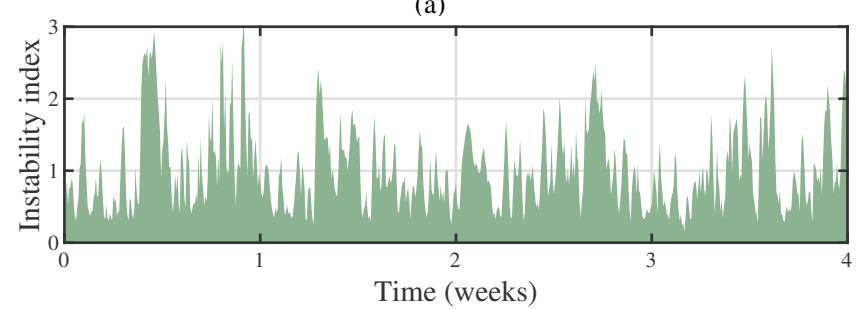

(b)

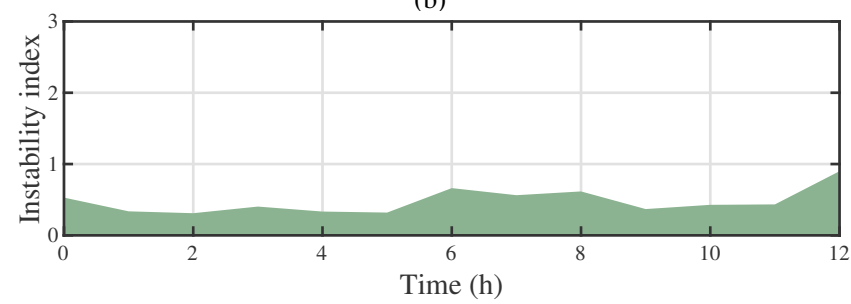

Fig. 10. Instability index for the station 62121: (a) Variation in 4 weeks. (b) Variation in 12 hours.

index variability, we apply it to modify only the subsequent three slots of the control horizon.

\section{Numerical case study}

Once we have introduced the dataset, let us now define the smart grid model employed in the numerical simulations. The smart grid has 500 users, 50 of which are active. We consider a total simulation time of 30 days and a control horizon of 
24 hours. The wind turbine is a NedWind- 40 with a rated power of $\omega_{\text {rated }}=500 \mathrm{~kW}$ and wind speed characteristic values $v_{\text {in }}=4 \mathrm{~m} / \mathrm{s}, v_{\text {rated }}=15 \mathrm{~m} / \mathrm{s}, v_{\text {out }}=25 \mathrm{~m} / \mathrm{s}$. The prosumers have a consumption curve that follows the average load hourly curve for UK households (without electric heating) [39]. The load curve has a daily average of $\sum e_{i}(h)=9.5 \mathrm{kWh}$ and presents a peak in the evening hours. The cost coefficient $K_{h}$ is $0.20 € / k W h$ for the daily hours (from 8:00 to 24:00) and $0.15 € / k W h$ at night (00:00 to 08:00). For the sake of simplicity, the global constraints are proportional with the average aggregate load. For the sake of simplicity, we assume for each prosumer a null maximum controllable energy demand, i.e., $\xi_{i}=0, \forall i \in \mathcal{N}$. The generation cost is supposed linear with a coefficient $\eta_{i}=0.04 € / k W h$; moreover, the maximum hourly dispatchable generation is $g_{i}^{\max }(h)=0.1 \mathrm{kWh}$ while the maximum generation is $\psi_{i}=0.8 H g_{i}^{\max }(h)$.

The storage devices are lithium-ion batteries, with a leakage rate $\alpha=0.90$, charging and discharging inefficiency equal to $\beta_{i}^{(+)}=0.99$ and $\beta_{i}^{(-)}=1.01, c_{i}=4 k W$ the battery capacity, $s_{i}^{\max }=0.5 c_{i} K W h$ the maximum charging rate and $c_{i}^{\text {initial }}=$ $q_{i}^{\min }=0.25 c_{i}$. Let us assume that all active users hold one dispatchable generation device and one energy storage device and that for the sake of simplicity, all the characteristics of these devices are identical. The computations for all the users are done in parallel. The gradient coefficient for Algorithm 1 is $\gamma=0.1$ and the termination criterion is $\left\|\boldsymbol{x}^{+}-\boldsymbol{x}\right\|_{2}^{2}+$ $\left\|\lambda^{+}-\lambda\right\|_{2}^{2} \leq 0.1$.

We added an additional day at the end of the simulation period with null energy demand and null wind power production. By adding this day, keeping energy in the battery would be a hidden cost for the prosumers. Therefore, we implicitly have that at the end of each simulation the state of charge of each battery equals the initial charge, thus, allowing us to compare fairly all the different set of simulations. In Fig. 12, we show the average state of charge for all active users $i \in \mathcal{S}$. From the figure, it is evident the highly cyclic pattern and that the state of charge at the end of the simulation period equal the initial one. Note that we also plotted the additional day to better show this behave.

Next, we perform an analysis from a stochastic point of view. Therefore, performing a single simulation for a defined set of data would not be significant. Hence, for each set of data, we repeat the simulation 50 times, which allows us to perform a more reliable analysis. In addition, in our analysis we aim at evaluating the savings obtained employing the proposed stochastic approach with respect to the standard expected value approach. However, before doing so, one could think of how big should the sampling size be to obtain an accurate approximate stochastic function. Hence, in the first case of analysis, we evaluate the results that a generic stochastic user obtains by increasing the number of samples. Let us remark that, for every sample size, we perform the simulation 50 times, and we evaluate the overall final results.

Figure 11(a) plots the standard deviation of the total costs that the stochastic user $i \in \mathcal{N}_{\mathcal{S}}$ pays to the grid. With the employed set of data, we obtain the convergence to the real stochastic value with approximately 200 samples; however, the total simulation time increases with the number of samples due

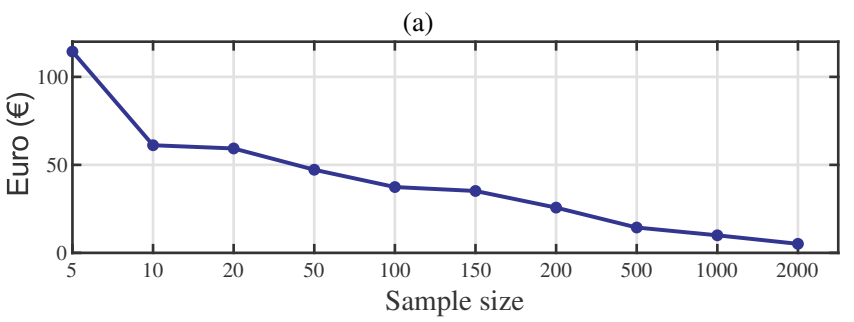

(b)

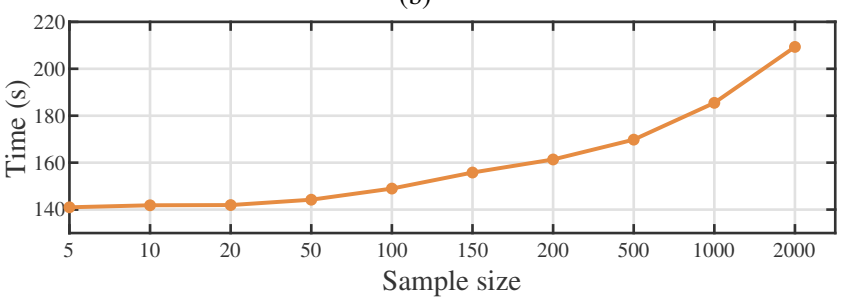

Fig. 11. Results for station 62121 as a function of the number of samples. (a) Final cost standard deviation. (b) Computation time.

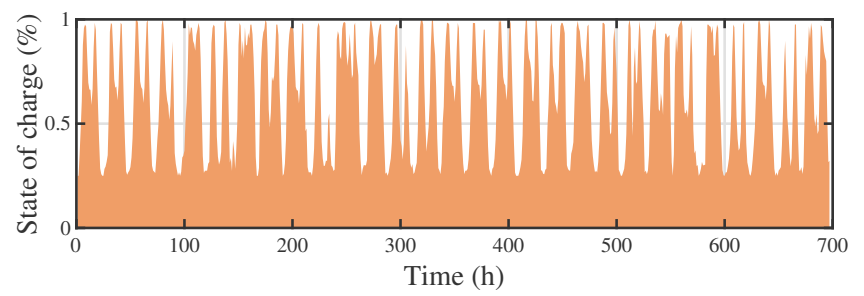

Fig. 12. Results for station 62121: Mean state of charge of all batteries

(a)

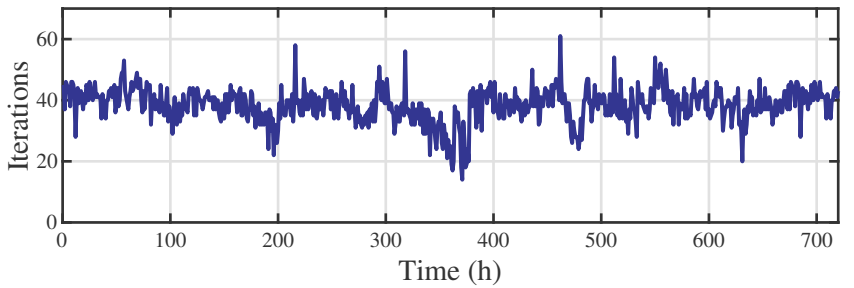

(b)

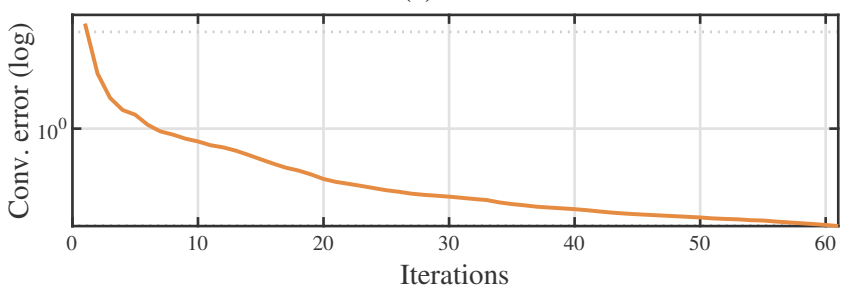

Fig. 13. Computational results for station 62121. (a) Number of iterations required for each time shift. (b) Iterations vs convergence plot.

to the increased complexity in the cost function.

Let us analyze the computational aspects of the proposed approach. Simulations are carried out in the MATLAB environment on a laptop equipped with a $1.3 \mathrm{GHz}$ Intel Core i5 CPU and 8 GB RAM. By employing the aforementioned termination criterion, and analyzing the results of all the stations, Algorithm 1 required, on average, approximately 40 iterations to converge. Moreover, the algorithm did not require more than 92 iterations with all the tested settings. Concerning the simulation time, on the aforementioned machine, 
TABLE III

ACTIVE USERS' STRATEGY (TEST USER)

\begin{tabular}{lll} 
Code & Test user strategy & Other users' \\
\hline Sim01 & Expected & 49 Expected \\
Sim02 & Historical PDF & 49 Expected \\
Sim03 & Forecast PDF & 49 Expected \\
Sim04 & Forecast PDF with inst. index & 49 Expected \\
Sim05 & Composed PDF & 49 Expected \\
Sim06 & Composed PDF with inst. index & 49 Expected \\
\hline
\end{tabular}

Algorithm 1 required, on average, 5.45 seconds to converge while the minimum and the maximum required time are 1.86 and 10.01 seconds, respectively. For instance, in Fig. 13 (a), we show the number of necessary iterations at each time shift of the simulation period, moreover, in Fig. 13 (b), we show the convergence to iteration plot for the time slot that required more iterations. Note that with 50 users, the chosen termination criterion is extremely severe.

The last case of analysis examines the performance of the proposed stochastic approach when different PDFs for the samples are employed. We analyze the results from the point of view of a single active user, named "test user". In Table III we show a recap for the different strategies adopted by the active user in the different sets of data. We calculate a naive cost for the test user by using the strategy labeled Sim01; this is the cost that the test user pays where he employs the expected value approach, together with all its active competitors.

In Fig. 14(a), we employ a Boxplot graph to show the savings or the losses that the test user obtains by adopting a different strategy to handle the uncertainty in wind power availability. In particular, using the strategy labeled Sim02, we show the results obtained with the mere use of the historical PDF and by neglecting the use of updatable forecasts; in this case, we obtain a loss with respect to the use of the forecast expected value. In addition, by the strategies labeled Sim03, Sim04, Sim05, and Sim06 we show how much the test user can save on average by adopting the proposed stochastic strategy. The simulations results show that the use of a better PDF, made by employing the instability index and/or by creating a composed PDF, increases the proposed approach performance.

Lastly, in Fig. 14(b) we show the total grid cost (the socalled welfare) when all the users employ a stochastic strategy, see Table IV for a recap of the strategies adopted by all the active user. The simulations show that, when all the active users consider a wind power PDF (i.e., in cases Sim13, Sim14, Sim15, Sim16), obviously the savings are lower than in case of a single stochastic user (i.e., in cases Sim03, Sim04, Sim05, Sim06), as showed by the comparison of Fig. 11 (a) with Fig. 11 (b). On the other hand, the results in Fig. 11 (b) confirm that there is a clear saving with respect to the expected value case. Finally, the higher savings are obtained in Sim16, i.e., when the composed PDF is used together with the instability index.

\section{CONCLUSION}

We presented a novel approach to implement demand side management in smart grids with uncertain wind power availability. The evidence from this study shows that in realistic
TABLE IV

ACTIVE USERS' STRATEGY (ALL PROSUMERS)

\begin{tabular}{ll} 
Code & All users strategy \\
\hline Sim12 & Historical PDF \\
Sim13 & Forecast PDF \\
Sim14 & Forecast PDF with inst. index \\
Sim15 & Composed PDF \\
Sim16 & Composed PDF with inst. index \\
\hline
\end{tabular}

(a)

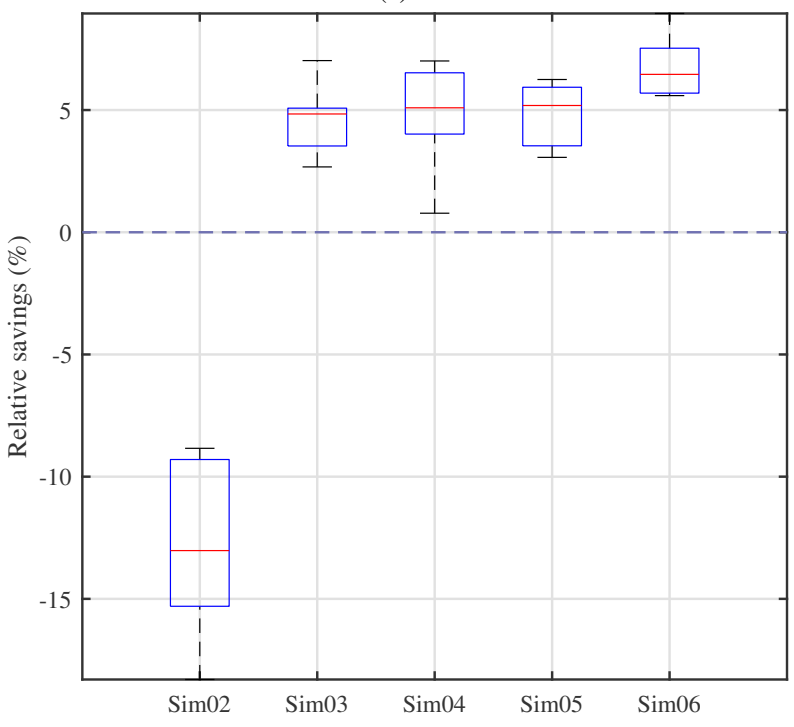

(b)

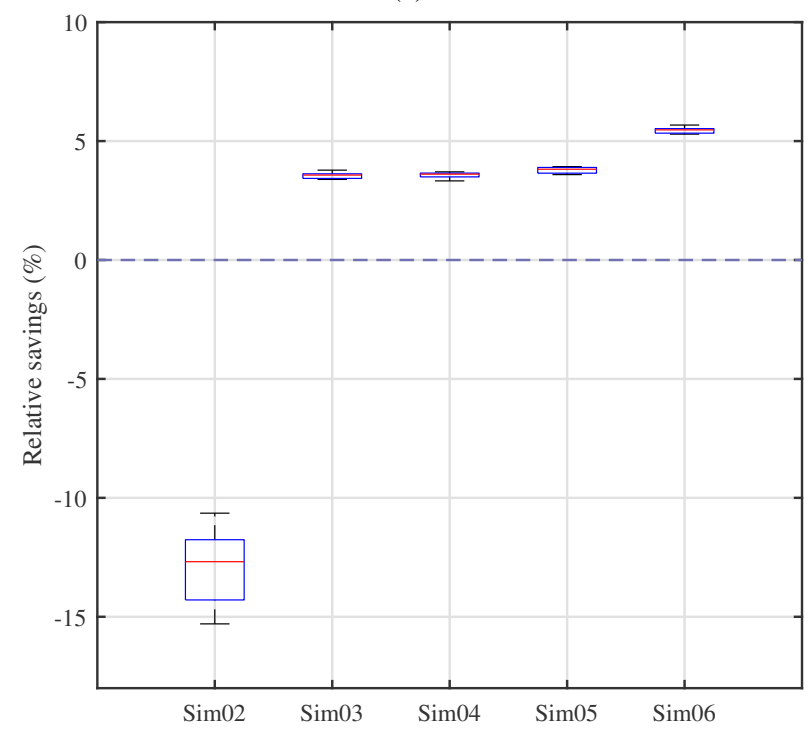

Fig. 14. Results for station 62121: (a) Boxplot of the test user savings with respect to the expected value strategy. (b) Boxplot of the total grid savings by employing a stochastic approach.

situations, considering different wind power availability scenarios increases the advantage of individual users against the competitors. In addition, we show that by including historical data and by taking into account the weather conditions with the proposed instability index, it is possible to improve the results significantly. Besides, the proposed approach is also able to improve the overall grid welfare.

Future work should focus on including the wind power availability in the global grid constraint, for instance by 
employing a penalty function or chance constraints. Moreover, the model could be enhanced by including other devices such as different kinds of controllable loads or individually owned energy sources, and integrating additional objective functions and technical constraints affecting the operations of system components.

\section{REFERENCES}

[1] M. L. Tuballa and M. L. Abundo, "A review of the development of smart grid technologies," Renew. Sust. Energ. Rev., vol. 59, pp. 710-725, 2016

[2] S. Kakran and S. Chanana, "Smart operations of smart grids integrated with distributed generation: A review," Renew. Sust. Energ. Rev., vol. 81, pp. 524-535, 2018.

[3] A. Ouammi, H. Dagdougui, and R. Sacile, "Optimal control of power flows and energy local storages in a network of microgrids modeled as a system of systems," IEEE Trans. Control Syst. Technol., vol. 23, no. 1, pp. 128-138, 2014.

[4] R. Carli, M. Dotoli, and R. Pellegrino, "A hierarchical decision-making strategy for the energy management of smart cities," IEEE Trans. Autom. Sci. Eng., vol. 14, no. 2, pp. 505-523, 2016.

[5] F. Dörfler, S. Bolognani, J. W. Simpson-Porco, and S. Grammatico, "Distributed control and optimization for autonomous power grids," in 2019 18th European Control Conference (ECC). IEEE, 2019, pp. 24362453.

[6] S. Maharjan, Q. Zhu, Y. Zhang, S. Gjessing, and T. Başar, "Demand response management in the smart grid in a large population regime," IEEE Trans. Smart Grid, vol. 7, no. 1, pp. 189-199, 2015.

[7] I. Atzeni, L. G. Ordóñez, G. Scutari, D. P. Palomar, and J. R. Fonollosa "Demand-side management via distributed energy generation and storage optimization," IEEE Trans. Smart Grid, vol. 4, no. 2, pp. 866-876, 2012.

[8] R. Carli and M. Dotoli, "Decentralized control for residential energy management of a smart users' microgrid with renewable energy exchange," IEEE/CAA Journal of Automatica Sinica, vol. 6, no. 3, pp. 641-656, 2019.

[9] R. Estrella, G. Belgioioso, and S. Grammatico, "A shrinking-horizon, game-theoretic algorithm for distributed energy generation and storage in the smart grid with wind forecasting," IFAC-PapersOnLine, vol. 52, no. 3, pp. 126-131, 2019.

[10] H. Wu, M. Shahidehpour, and A. Al-Abdulwahab, "Hourly demand response in day-ahead scheduling for managing the variability of renewable energy," IET Gener. Transm. Distrib., vol. 7, no. 3, pp. 226-234, 2013.

[11] C. A. Hans, P. Sopasakis, J. Raisch, C. Reincke-Collon, and P. Patrinos, "Risk-averse model predictive operation control of islanded microgrids," IEEE Trans. Control Syst. Technol., 2019.

[12] R. Jabr and B. C. Pal, "Intermittent wind generation in optimal power flow dispatching," IET Gener. Transm. Distrib., vol. 3, no. 1, pp. 66-74, 2009.

[13] F. Yao, Z. Y. Dong, K. Meng, Z. Xu, H. H.-C. Iu, and K. P. Wong, "Quantum-inspired particle swarm optimization for power system operations considering wind power uncertainty and carbon tax in australia," IEEE Trans. Ind. Informat., vol. 8, no. 4, pp. 880-888, 2012.

[14] P. P. Biswas, P. Suganthan, and G. A. Amaratunga, "Optimal power flow solutions incorporating stochastic wind and solar power," Energy Convers. Manag., vol. 148, pp. 1194-1207, 2017.

[15] H. Quan, D. Yang, A. M. Khambadkone, and D. Srinivasan, "A stochastic power flow study to investigate the effects of renewable energy integration," in 2018 IEEE Innovative Smart Grid Technologies-Asia (ISGT Asia). IEEE, 2018, pp. 19-24.

[16] G. Aghajani, H. Shayanfar, and H. Shayeghi, "Demand side management in a smart micro-grid in the presence of renewable generation and demand response," Energy, vol. 126, pp. 622-637, 2017.

[17] Y. Kun, K. Zhang, Y. Zheng, L. Dawei, W. Ying, and Y. Zhenglin, "Irregular distribution of wind power prediction," Journal of Modern Power Systems and Clean Energy, vol. 6, no. 6, pp. 1172-1180, 2018.

[18] W. Ko, D. Hur, and J.-K. Park, "Correction of wind power forecasting by considering wind speed forecast error,' Journal of International Council on Electrical Engineering, vol. 5, no. 1, pp. 47-50, 2015.

[19] S. Pazouki, M.-R. Haghifam, and A. Moser, "Uncertainty modeling in optimal operation of energy hub in presence of wind, storage and demand response," International Journal of Electrical Power \& Energy Systems, vol. 61, pp. 335-345, 2014
[20] K. Afshar and A. Shokri Gazafroudi, "Application of stochastic programming to determine operating reserves with considering wind and load uncertainties,' Journal of Operation and Automation in Power Engineering, vol. 1, no. 2, pp. 96-109, 2007.

[21] Y. Zheng, S. Li, and R. Tan, "Distributed model predictive control for on-connected microgrid power management," IEEE Trans. Control Syst. Technol., vol. 26, no. 3, pp. 1028-1039, 2017.

[22] A.-H. Mohsenian-Rad, V. Wong, J. Jatskevich, R. Schober, and A. LeonGarcia, "Autonomous demand side management based on gametheoretic energy consumption scheduling for the future smart grid," IEEE Trans. Smart Grid, vol. 1, no. 3, pp. 320-331, 2010.

[23] M. S. Gao and H. Jiang, "A dispatch algorithm for smart grid with wind generation," in Applied Mechanics and Materials, vol. 704. Trans Tech Publ, 2015, pp. 186-189.

[24] A. Ghaffari, M. Krstić, and S. Seshagiri, "Power optimization and control in wind energy conversion systems using extremum seeking," IEEE Trans. Control Syst. Technol., vol. 22, no. 5, pp. 1684-1695, 2014.

[25] J. A. Carta, P. Ramirez, and S. Velazquez, "A review of wind speed probability distributions used in wind energy analysis: Case studies in the canary islands," Renew. Sust. Energ. Rev., vol. 13, no. 5, pp. 933955, 2009.

[26] Nedwind-40 technical data sheet. NedWind Rhenen bV. [Online]. Available: "https://www.windsofchange.dk/downloads.php?a=66\&t=1\& $\mathrm{f}=$ Nedwind.pdf'

[27] J. Hetzer, C. Y. David, and K. Bhattarai, "An economic dispatch model incorporating wind power," IEEE Trans. on Energy Convers., vol. 23, no. 2, pp. 603-611, 2008 .

[28] R. B. Schinazi, Transformations of Random Variables and Random Vectors. Springer, 2012.

[29] T. Iizaka, T. Jintsugawa, H. Kondo, Y. Nakanishi, Y. Fukuyama, and H. Mori, "A wind power forecasting method and its confidence interval estimation," Electrical Engineering in Japan, vol. 186, no. 2, pp. 52-60, 2014.

[30] P. Pinson and G. Kariniotakis, "On-line assessment of prediction risk for wind power production forecasts," Wind Energy: An International Journal for Progress and Applications in Wind Power Conversion Technology, vol. 7, no. 2, pp. 119-132, 2004.

[31] R. T. Clemen and R. L. Winkler, Calibrating and combining precipitation probability forecasts. Springer, 1987.

[32] G. Scutari, D. P. Palomar, F. Facchinei, and J.-s. Pang, "Convex optimization, game theory, and variational inequality theory," IEEE Signal Process. Mag., vol. 27, no. 3, pp. 35-49, 2010.

[33] G. Belgioioso and S. Grammatico, "Projected-gradient algorithms for generalized equilibrium seeking in aggregative games are preconditioned forward-backward methods," in 2018 European Control Conference (ECC). IEEE, 2018, pp. 2188-2193.

[34] A. Shapiro, "Stochastic programming approach to optimization under uncertainty," Mathematical Programming, vol. 112, no. 1, pp. 183-220, 2008.

[35] S. Kim, R. Pasupathy, and S. G. Henderson, A guide to sample average approximation. Springer, 2015.

[36] Meteorological assimilation data ingest system. National Oceanic and Atmospheric Administration. [Online]. Available: "www.madis.ncep. noaa.gov"

[37] Storm glass - the global weather forecast api. Människa Maskin AB [Online]. Available: "www.stormglass.io"

[38] Weather api - historical weather api. Weatherbit LCC. [Online]. Available: www.weatherbit.io/

[39] Household electricity survey. Department of Energy and Climate Change. [Online]. Available: "www.gov.uk/government/publications/ household-electricity-survey--2"

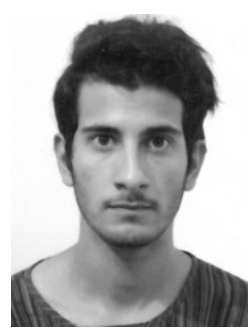

Paolo Scarabaggio (S'20) received the B.Sc. degree in Mechanical Engineering in 2017 and the M.Sc. degree in Management Engineering in 2019 from the Politecnico di Bari, Bari, Italy. In 2019, he was a visiting student at the Delft Center for Systems and Control, TU Delft, The Netherlands.

$\mathrm{He}$ is currently working toward the Ph.D. degree in the Dept. of Electrical and Information Engineering of the same university under the supervision of Prof. Engr. Mariagrazia Dotoli.

His research interests include modeling, optimization, and distributed control of energy and complex networked systems. 


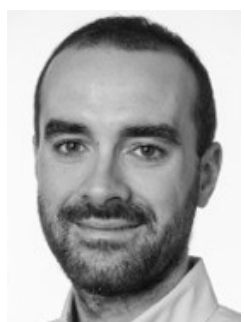

Sergio Grammatico (M'16, SM'19) is an Associate Professor in the Delft Center for Systems and Control, TU Delft, The Netherlands. Born in Italy, in 1987, he received the Bachelor degree in Computer Engineering, the Master degree in $\mathrm{Au}-$ tomatic Control Engineering, and the Ph.D. degree in Automatic Control, all from the University of Pisa, in 2008, 2009, and 2013 respectively. He also received a Master degree in Engineering Science from the Sant'Anna School of Advanced Studies, Pisa, the Italian Superior Graduate School (Grande École) for Applied Sciences, in 2011. In 2012, he visited the Department of Electrical and Computer Engineering at the University of California at Santa Barbara; in 2013-2015, he was a post-doctoral Research Fellow in the Automatic Control Laboratory at ETH Zurich; in 2015-2017, he was an Assistant Professor in the Control Systems group at TU Eindhoven.

His research revolves around game theory, optimization and control for complex multi-agent systems, with applications in power grids and automated driving. He was awarded 2013 and 2014 TAC Outstanding Reviewer and he was recipient of the Best Paper Award at the 2016 ISDG International Conference on Network Games, Control and Optimization.

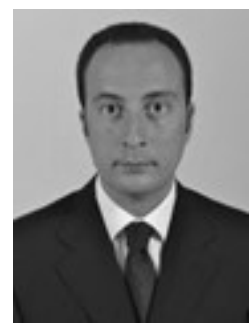

Raffaele Carli (M'17) received the Laurea degree in Electronic Engineering with honours in 2002 and the Ph.D. in Electrical and Information Engineering in 2016, both from Politecnico di Bari, Italy. From 2003 to 2004, he was a Reserve Officer with Italian Navy. From 2004 to 2012, he worked as System and Control Engineer and Technical Manager for a space and defense multinational company.

Dr. Carli is currently a research fellow at Politecnico di Bari, and his research interests include the formalization, simulation, and implementation of decision and control systems, as well as modeling and optimization of complex systems. He was member of the International Program Committee of 20+ international conferences and guest editor for special issues on international journals. He is author of $60+$ printed international publications.

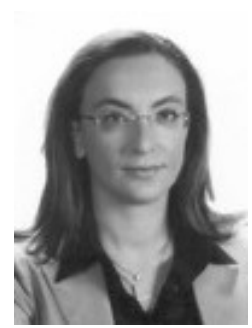

Mariagrazia Dotoli (M'96, SM'12) received the Laurea degree in Electronic Engineering with honours in 1995 and the Ph.D. in electrical engineering in 1999 from Politecnico di Bari (Italy).

She has been a visiting scholar at the Paris 6 University and at the Technical University of Denmark. She is expert evaluator of the European Commission since the 6th Framework Programme. She is a Full Professor in Automatic Control at Politecnico di Bari, which she joined in 1999. She has been the Vice Rector for research of Politecnico di Bari and a member elect of the Academic Senate. She is currently the coordinator of the Industry 4.0 PhD Program of Politecnico di Bari and University of Bari (Italy). Her research interests include modeling, identification, management, control and diagnosis of discrete event systems, manufacturing systems, logistics systems, traffic networks, smart grids and networked systems.

Prof. Dotoli was co-chairman of the Training and Education Committee of ERUDIT, the European Commission network of excellence for fuzzy logic and uncertainty modeling in information technology, and was key node representative of EUNITE, the EUropean Network of excellence on Intelligent Technologies. She is the Editor in Chief of the IEEE SYSTEMS MAN AND CYBERNETICS SOCIETY E-NEWSLETTER, and an Associate Editor of the IEEE TRANS. ON AUTOMATION SCIENCE AND ENGINEERING, of the IEEE TRANS. ON SYSTEMS, MAN, AND CYBERNETICS and of the IEEE TRANS. ON CONTROL SYSTEMS TECHNOLOGY. She is the Program chair of the 2020 IEEE Conference on Automation Science and Engineering and the General chair of the 29th Mediterranean Conference on Control and Automation. She was the Program co-chair of the 2017 IEEE Conference on Automation Science and Engineering, the Workshop and Tutorial chair of the 2015 IEEE Conference on Automation Science and Engineering, the Special Session co-chair of the 2013 IEEE Conference on Emerging Technology and Factory Automation and chair of the National Committee of the 2009 IFAC Workshop on Dependable Control of Discrete Systems. She was member of the International Program Committee of $80+$ international conferences. She is author of $200+$ publications, including 1 textbook (in Italian) and 70+ international journal papers. 